\title{
DEMOGRAFÍA DIFERENCIAL DE LA MINORÍA MORISCA: UNA APORTACIÓN A SU ESTUDIO*
}

\author{
María del Carmen Ansón Calvo**
}

\section{DATOS DE PARTIDA Y METODOLOGÍA USADA EN SU EXPLOTACIÓN}

Los mandatos del Concilio de Trento (1545-1563) relativos a la obligación, por parte de los párrocos, de llevar puntualmente los Cinco Libros ${ }^{1}$, desafortunadamente comienzan a cumplirse en algunas partes de Aragón en tiempos muy próximos a la fecha de la expulsión de los moriscos de España. Ello originó, en el caso de Torrellas, pueblo de Aragón del Señorío de Villahermosa poblado totalmente por moriscos elegido para este estudio, que sólo se recogieran datos de bautismos desde el año 1596 (5/7/1596) y de matrimonios desde el mismo año (22/6/1596). Sin embargo, las actas de difuntos de jóvenes y mayores comenzaron a recogerse desde algunos meses antes $(2 / 2 / 1596)$ dentro del mismo año. Desde este punto de vista, el año 1596, al no estar completo, no nos será útil para determinar la tendencia de evolución de los bautismos antes de la expulsión. En sentido semejante, como la expulsión se produce en el caso de Torrellas en agosto del año 1610, como lo demuestra que el último bautismo recogido en el libro pertinente tiene fecha de 28 de julio de dicho año, en forma semejante a lo que sucedía en el año 1596, tampoco los datos del año 1610 serán útiles para determinar la tendencia de evolución de los bautismos antes de la expulsión. De forma paralela, el acta de matrimonio de fecha más avanzada es del 30 de junio de 1610 y la última de entierro del 12 de agosto del mismo año. Nos quedarán, pues, trece años completos de datos útiles de bau-

\footnotetext{
* Trabajo presentado en el VII Congreso de Demografía Histórica (ADEH). Granada, mayo 2004.

** Universidad de Oviedo.

1. L. Belluga, Concilii Tridentini, Roma, 1773.
} 
tismos, matrimonios y entierros de mayores, intervalo temporal pequeño para obtener solventemente determinado tipo de informaciones demográficas. De cualquier modo, la situación es la que es y con ella habremos de trabajar para colaborar al conocimiento demográfico de esta minoría ${ }^{2}$.

Entre las dos fechas antes citadas encontramos 965 actas útiles de bautismos (hay una más, pero lleva una fecha del año 1587, probablemente debida a un error del párroco), 266 actas útiles de matrimonios y 274 actas útiles de defunciones (hay 6 más en las que el párroco correspondiente no consignó la fecha). Una vez transcritas estas Actas Sacramentales las introdujimos en una base de datos empleando un programa de biblioteca llamado File Maker Pro, Versión 6. Las informaciones que se previó pudieran recogerse fueron las mismas que en el caso del Archivo Parroquial de San Pablo de Zaragoza ${ }^{3}$ pues, con ellas, obtuvimos bastantes resultados de interés demográfico y de estructuras y comportamientos sociales. Dado que teníamos experiencia en el manejo de este tipo de datos, consideramos que no era absurdo emplearla en este caso, cambiando, como es lógico, "la forma de relacionarlos", ya que los métodos de trabajo de una base de datos, comercial son característicos de la misma y tendríamos que adaptarnos a ellos para obtener las informaciones que buscábamos.

Parece pertinente hacer aquí un pequeño comentario. Además del objetivo de obtener cuantas más informaciones de todo tipo que pudiésemos sobre la población de "cristianos nuevos" de Torrellas, teníamos el propósito de explorar qué tipo de dificultades podía presentar el uso de un programa comercial de base de datos en una investigación basada en los datos de los archivos parroquiales de bautismos, matrimonios y defunciones. Los programas de bases de datos están construidos para relacionar entre sí informaciones de tipo equivalente procedentes de muchos casos, pero no para relacionar las informaciones diferentes de un solo caso. Como es evidente, esta situación plantea "algunas imposibilidades" en la obtención de determinadas informaciones, especialmente si se desea utilizar sus potencialidades internas para generar fi-

2. Citas documentales comprendidas en el texto:

A(rchivo) D(iocesano) de Tarazona.- Actas Sacramentales de Bautismos, Matrimonios y Entierros de la Parroquia de Torrellas. Años 1596 a 1610.

A(rchivo) D(iocesano) de Tarazona.- Actas Sacramentales de Bautismos, Matrimonios y Entierros de la Parroquia de Santa Cruz de Moncayo. Años 1587 a 1610.

A(rchivo) H(istórico) M(unicipal) de Torrellas.-Libro del Justicia.

A(rchivo) H(istórico) N(acional).- Sección Inquisición. Documentación Inquisitorial.- Legajos 988, 989, 990 y 991 .

A(rchivo) H(istórico) No(tarial) de Tarazona.- Protocolos de los notarios Tristán Maestro, Gabriel Maestro y Gabriel de Yunez (años 1547 a 1606).

3. M.C. ANsón CAlvo, Demografía y sociedad urbana en la Zaragoza del siglo XVII, Zaragoza, 1977, pp. 31-35. 
chas de familia. Al final, ha sido posible hacerlo utilizando un método de trabajo semi-automático. De hecho, hemos llegado a construir bastantes familias abiertas mezclando los datos de bautismos y los de matrimonios. Lo que sí es posible es obtener, a través de "informes", listados y recuentos de todo tipo sobre datos análogos.

Por otro lado, como uno de los propósitos de esta investigación era hallar informaciones que nos permitieran aportar alguna información sobre la cuestión, para nosotros bastante crucial, de si las comunidades de moriscos en Aragón se "diferenciaban o no" de las comunidades de cristianos viejos, como disponemos de los datos equivalentes de poblaciones de este último tipo para la misma época, que, en conjunto, sumaban 6.111 habitantes y formaban parte del llamado Campo de Cariñena ${ }^{4}$, distando unos $100 \mathrm{Km}$ de Torrellas, en esta aportación continuamente compararemos los comportamientos de ambos colectivos, el de cristianos nuevos y el de cristianos viejos. La población del lugar de Torrellas (Comarca de Tarazona) en la fecha de la expulsión de los moriscos, año 1610, contaba con 2.040 habitantes (408 casas). La tabla 1 recoge los datos de bautismos, matrimonios y entierros o defunciones de mayores del lugar de Torrellas entre los años 1596 y 1610.

Tabla 1. Números anuales de bautismos, matrimonios y defunciones de mayores de Torrellas.

\begin{tabular}{lccccc}
\hline AÑO & BAU. V & BAU. H & BAU. T & MATR. & ENT.(M) \\
\hline 1596 & 23 & 11 & 34 & 15 & 20 \\
1597 & 33 & 25 & 58 & 22 & 21 \\
1598 & 31 & 29 & 60 & 25 & 18 \\
1599 & 36 & 23 & 59 & 12 & 18 \\
1600 & 42 & 36 & 78 & 11 & 11 \\
1601 & 37 & 30 & 67 & 19 & 11 \\
1602 & 47 & 38 & 84 & 23 & 33 \\
1603 & 37 & 22 & 59 & 18 & 15 \\
1604 & 50 & 32 & 82 & 19 & 30 \\
1605 & 46 & 36 & 82 & 19 & 15 \\
1606 & 52 & 32 & 84 & 19 & 21 \\
1607 & 19 & 16 & 35 & 16 & 20 \\
1608 & 39 & 23 & 62 & 12 & 19 \\
1609 & 27 & 34 & 61 & 15 & 21 \\
1610 & 37 & 23 & 60 & 15 & 1 \\
\hline
\end{tabular}

4. M.C. Ansón CALVO, «Un ejemplo de demografía diferencial en Aragón: Campo y ciudad en el siglo XVII», Boletín de la Asociación de Demografía Histórica, VIII-3 (1990), pp. 21-65.

5. J. Reglá, Estudios sobre los moriscos, Anales de la Universidad de Valencia, Filosofía y Letras, Cuaderno VI, V. XXXVII, 1963-1964, p. 470. 


\section{BAUTISMOS, MATRIMONIOS Y DEFUNCIONES DE MAYORES}

\section{BAUTISMOS Y MATRIMONIOS}

La figura 1 recoge las evoluciones con el tiempo de los números anuales de bautismos y de matrimonios de Torrellas entre los años 1597 y 1609. Con los de bautismos, extrapolándolos al año 1610, nos aparece para la comunidad morisca de Torrellas una Tasa Bruta de Natalidad (TBN) de 32,43 nacimientos (bautismos) por mil habitantes. En la primera mitad del siglo XVII, los siete pueblos del Campo de Cariñena, con una población de 6.111 habitantes en el año 1646, presentaban una TBN de 37,00 nacimientos por mil habitantes ${ }^{6}$. Este par de valores parecen contradecir todos los comentarios de los autores de la época anterior a la expulsión 7 que asignaban a los moriscos una natalidad que crecía vertiginosamente.

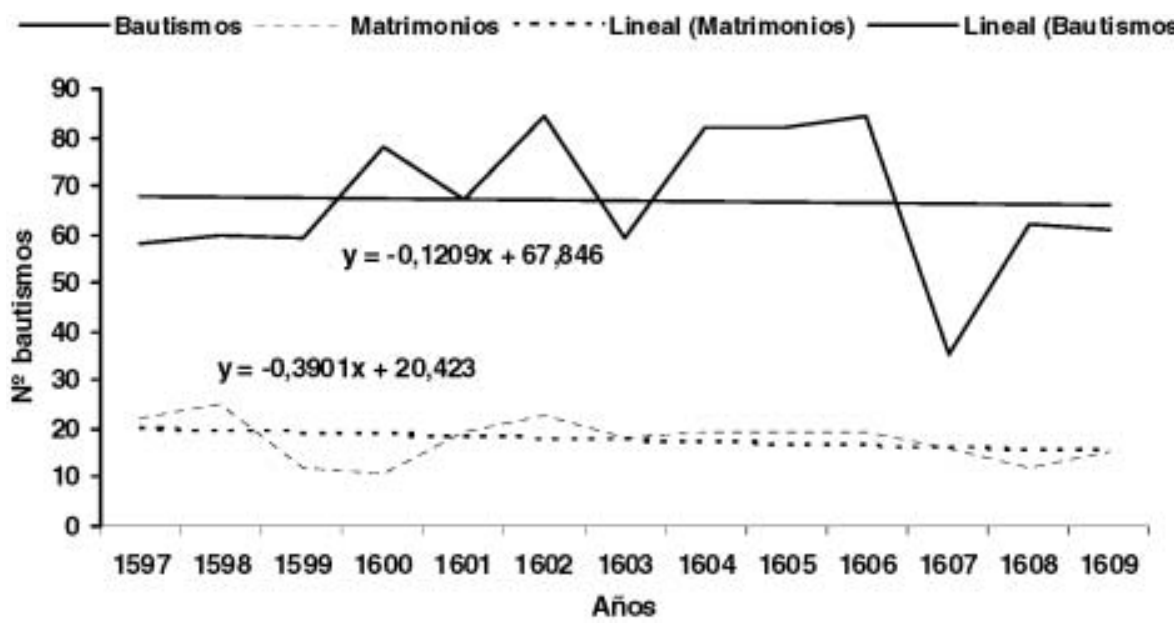

Figura 1. Números anuales de bautismos y matrimonios en Torrellas.

Mediante los datos de números anuales de matrimonios del mismo periodo (1597 a 1609) se puede extrapolar el valor correspondiente al año 1610 con el que se deduce, para Torrellas, una Tasa Bruta de Nupcialidad (TBNU) de

6. M.C. AnSÓN CALVO, «Un ejemplo de demografía diferencial en Aragón», p. 58.

7. P. AzNAR CARDONA, Expulsión justificada de los moriscos españoles, Huesca, 1612, vol. II, p. 35 y ss.; M. de Guadalajara, Memorable expulsión y justísimo destierro de los moriscos de España, Huesca, 1613, p. 97. 
7,33 bodas por mil habitantes. En la primera mitad del siglo XVII, los siete pueblos del Campo de Cariñena, cuya población en el año 1646 hemos señalado antes, presentaban una TBNU de 9,35 bodas por mil habitantes. En el caso de los matrimonios, la comunidad morisca de Torrellas en 1610 tenía pues una nupcialidad "menor" que la del Campo de Cariñena en el año 1646.

Observando las "representaciones lineales" de los datos de bautismos y de matrimonios de la figura 1 , se constata que, ambas, tienen pendiente negativa, mayor la de las matrimonios que la de los bautismos. Ello indica que, muy posiblemente, la población de Torrellas fue disminuyendo al acercarse la fecha de su expulsión (datos de bautismos), debido muy posiblemente a que tuvieron noticias de esta decisión mientras se gestaba.

El resultado hallado en relación con la natalidad de la población morisca de Torrellas no nos pareció creíble en principio, pues su TBN es bastante baja, aunque no imposible. Hay varias causas externas que pudieran dar lugar al cálculo de una tasa de natalidad baja y, de ellas, la primera pudiera ser que la cifra de población utilizada para calcularla haya sido más elevada que la real. Según datos documentales, en el año 1610 fueron expulsados los moriscos que vivían en 408 casas (2.040 moriscos a 5 pobladores por casa), según los contaban las tropas del marqués de Aytona ${ }^{8}$. Si se toma como totalmente correcta esta cifra conduce, como hemos visto, a una TBN de 32,43 nacimientos por mil habitantes, baja para una población que se supone expansiva, lo cual plantea la disyuntiva de que, o bien el recuento de los expulsados fue muy "por exceso", o que las percepciones cualitativas de los escritores de la época sobre la elevada natalidad de las familias moriscas, basadas fundamentalmente en la juventud de las moriscas que accedían al matrimonio, eran erróneas ${ }^{9}$. Respecto a la primera posibilidad, podría ser cierta si se admitiera que, en Torrellas, además de su población, fueron concentrados moriscos de "zonas próximas" para llevar a cabo su expulsión conjunta. Si ello se llevó a cabo de esta forma, los moriscos expulsados "verdaderamente de Torrellas" quizás fueron menos que los correspondientes a las 408 casas contadas por los oficiales del marqués de Aytona, por lo que, si pudiéramos emplear la cifra real en el cálculo, la TBN correspondiente a la población de Torrellas "subiría" de valor. Sin embargo, tal concentración no se produjo, pues el párroco de Torrellas, D. Miguel de Yerga, en el libro de casados, el 12 de agosto de 1610 hace una anotación en la que dice que "fueron expulsos los moriscos de esta villa, salieron 2.012 sin los niños y niñas de teta" y el mismo párroco, en el libro de bautismos, en fecha 12 de agosto de 1610, escribe una nota que dice: "salieron desterrados los moriscos de la villa, 2.000 personas y los niños de teta de 2 años abajo" (A. D. de Tarazona, Libro I de Casados y Bautizados de la Pa-

8. J. ReGLÁ, Estudios sobre los moriscos, p. 470.

9. P. AZnAR CARDONA, op. cit., vol. II, pp. 53 y 113-116. 
rroquia de San Martín de Torrellas). Así pues, la cifra de 2.040 habitantes que hemos utilizado para el año 1610 parece ser correcta. Confirmando lo anterior, con los datos de intervalos entre bautismos sucesivos para familias que tuvieron más de un hijo, datos que, después, comentaremos, se puede calcular que, probablemente, murieron 73 niños de los nacidos en los 24 meses previos a la expulsión. Como estos suman unos 120, los que quedarían vivos serían unos 50, es decir, con gran aproximación, el número de los niños de " 2 años abajo" que refiere el párroco de Torrellas. Las cifras dadas por el párroco de Torrellas y por los oficiales del marqués de Aytona concuerdan bien entre sí.

Otra de las causas de la baja TBN calculada podría estar en que los números anuales de bautismos recogidos en los libros parroquiales fueran "menos" que los debidos a los niños realmente nacidos, a causa de ocultaciones por razones de índole religiosa. En la tabla 1, además de los números anuales de bautismos totales, se recogen los datos correspondientes a los bautismos desglosados por sexos. Con el fin de encontrar algún indicio sobre si tal cosa sucedió realmente en Torrellas, utilizando dichos datos para determinar la relación de bautizos de varones a los de hembras, encontramos que, en Torrellas, empleando los datos de los años 1597 a 1609, dicha relación fue de 1,32 niños por cada niña. En el Campo de Cariñena fue, entre los años de 1600 a 1650 , de $1,06^{10}$ y esta misma relación, 1,06, es la encontrada para Zaragoza entre los años 1600 y $1650^{11}$. Este valor era, prácticamente, el que a mediados del siglo XX también se daba en España ${ }^{12}$.

En principio, pensamos que de existir ocultaciones serían de nacimientos de "niños", en función de la práctica musulmana de la circuncisión de los varones, proceder que corresponde, en la práctica, a un "bautismo musulmán" pero, lo cierto es que, si la hubo, la ocultación de bautismos no debió de venir por el lado de los niños, porque los párrocos estaban "muy al tanto" de los bautismos de varones y porque los niños eran "muy valiosos" para las familias. El control en el bautismo de niños en Torrellas debió de ser muy fuerte, en especial sobre la práctica de la circuncisión, como se deduce de ciertas noticias documentales. Así, por ejemplo, en los protocolos notariales del notario Tristán Maestro del año 1565 (A. H. No. de Tarazona) hemos encontrado un caso en que la familia solicita la elaboración de un acta notarial en la que se hacía constar que el niño había sido "retajado" por mano de un cirujano de Tarazona como consecuencia de una enfermedad. Otras veces, es el propio párroco el que anota en el libro de bautismos (Acta de bautismo del 20 de junio de 1603) que "este niño nació retajado" y hay otras en que aparece, diciendo lo mismo, pero

10. M.C. AnSÓN CALVO, «Un ejemplo de demografía diferencial en Aragón», p. 58.

11. M.C. Ansón CALVo, Demografía y sociedad urbana en la Zaragoza del siglo XVII, p. 76.

12. A. Arbelo Curbelo, Sanidad Infantil en España, Madrid, 1971, p. 20. 
con la firma del cirujano. Así pues, en sentido contrario a lo antes aducido, tenemos que concluir que, muy probablemente, la causa del "elevado" valor de la relación varón / hembra para los bautismos de Torrellas podría estar en la ocultación, o "no práctica", del bautismo de niñas y no de niños, por no pensar en prácticas más "siniestras". El valor de una mujer en la Edad Moderna era muy inferior al de un hombre y menos todavía en la comunidad morisca, y, desde luego, en una familia campesina pobre el valor de una niña era muy pequeño ya que, en cierto sentido, era más una carga que una bendición, pues no iba a proporcionar brazos para trabajar la tierra en el futuro y, para casarla, requería una dote difícil de aportar por la familia. No podemos asegurar que en Torrellas, a finales del siglo XVI y comienzos del XVII, se hubiese practicado una ocultación de nacimientos de niñas a fin de evitar su bautismo, pero los datos aportados son inquietantes y sugieren que quizás hubo "algo más" que simples ocultaciones y en las que posiblemente se tuvo que contar con la complicidad de las comadres.

Además de estas razones, se podrían aducir también otras para explicar el elevado valor de la relación niño a niña en el nacimiento y entre ellas la más fácil de admitir es que, lo hallado, quizás fuera una consecuencia del pequeño número de años acumulados, cosa que podría haber conducido, por pura estadística, a una situación aparentemente anormal de esta relación. Sin embargo, calculadas las relaciones individuales niño/niña para los datos de los 13 años implicados, sólo en un año, 1609, la relación varón a hembra resulta estar fuera de la horquilla que la desviación estándar de la media $(1,34 \pm 0,27)$ de dichos datos, pues vale 0,79. El valor de las relaciones varón a hembra de la población de cristianos nuevos de Torrellas es, pues, sistemáticamente mayor que en las poblaciones de cristianos viejos del Campo de Cariñena donde la media es de 1,06. Este valor, en el caso de la población del Campo de Cariñena, procede de los valores de bautismos de niños y niñas correspondientes a 46 años, 1600 a 1645, en los que, en todos los casos, sus relaciones fueron inferiores a 1,34, o sea, sistemáticamente inferiores al promedio correspondiente a los datos de Torrellas. La curiosidad y singularidad de lo hallado nos llevó a comparar los datos de bautismos del pueblo morisco de Torrellas con los que hemos obtenido del vaciado de las Actas Sacramentales de otro pueblo, también de moriscos, distante sólo $2 \mathrm{Km}$ de Torrellas, Santa Cruz de Moncayo, de 650 habitantes (130 casas a 5 pobladores por casa $)^{13}$. Sus libros parroquiales de bautismos, que comienzan en 1587 y terminan en 1610, nos han permitido contabilizar 263 niños y 237 niñas entre los 500 bautizados habidos, es decir, los nacimientos se producen con una relación varón a hembra de

13. A. Ubieto Arteta, Historia de Aragón: los pueblos y los despoblados, tomos I, II y III, Zaragoza, 1985 , p. 1147. 
1,11, valor también mayor que el encontrado en el Campo de Cariñena, pero bastante inferior al hallado para la población de Torrellas. Es interesante indicar que lo hallado en el caso de Torrellas no se reproduce tampoco en otras comunidades moriscas de la época. Así, en la comunidad morisca andaluza de Comares ${ }^{14}$, los datos aportados por este autor sobre bautismos de hijos e hijas entre los años 1547 a 1570, en los que la población de moriscos era de 350 hijos y 348 hijas, conducen a una relación de bautismos varones a hembras de 1,01. Los datos aportados son por quinquenios y su suma es la que hemos dado aquí. El problema queda así y requerirá muchos más datos para encontrar una respuesta definitiva a la desproporcionada relación niños/niñas en el bautismo encontrada para Torrellas, pues otras razones que se podrían aducir serían más complejas de admitir, ya que parece ilógico a todas luces suponer una estructura genética especial en los moriscos que hiciera fallar las puras "formas de mezcla" de los cromosomas X e Y que operan en el común de los humanos. Así pues, la ocultación de nacimientos de niñas muy posiblemente pudo operar en la comunidad de Torrellas, cosa que, parece, no sucedía en las otras dos comunidades de moriscos citadas y tampoco en los colectivos del Campo de Cariñena.

Los datos de números anuales de bautismos en Torrellas entre los años 1597 a 1609, a través de la recta de mínimos cuadrados, permiten el cálculo, por extrapolación, del "mejor valor de bautismos en el año 1610" (66,15 bautismos). Estos, utilizando la cifra de moriscos expulsados en este año (2.040 pobladores), son los que proporcionan la Tasa Bruta de Natalidad del 32,43 por mil, antes citada. Si supusiéramos que en la población de Torrellas la relación varón a hembra en el nacimiento fuera la misma que en el caso del Campo de Cariñena (1,06 niños por niña), podríamos calcular un "número total ficticio de nacimientos de niñas", 468, entre los años 1597-1609, que sumados a los 496 bautismos de niños darían una cifra 964 bautismos totales, en vez de los 872 a que dan lugar las 376 niñas computadas a partir de los datos originales. Con esta misma metódica se pueden calcular los números anuales de bautismos totales en cada uno de los años del periodo estudiado, conservando la proporción varón a hembra de 1,06. Con ellos, mediante una recta de mínimos cuadrados, sería posible extrapolar, de nuevo, un "mejor valor de bautismos en el año 1610". Este resulta ser de 73,17 bautismos, con el que se calcula una TBN de 35,87 nacidos por mil habitantes. Este valor, en principio, es más adecuado que el de 32,43 calculado a partir de los datos originales. Una TBN más cercana al 36 por mil es suficientemente representativa para la época estudiada, en función de los intervalos entre nacimientos sucesivos que, habitual-

14. J.J. BRAVO CARO, «La familia morisca a través de los registros parroquiales», en A. Temimi (ed.), Actes du VIIe Symposium International d'Etudes Morisques sur: Famille Morisque: Femmes et Enfants. Familia Morisca: Mujeres y Niños, Zaghouan, 1997, p. 35. 
mente, se daban en el Campo de Cariñena ${ }^{15}$ y que, para el caso de la población de Torrellas, no son muy diferentes, como veremos más adelante.

\section{ENTIERROS DE MAYORES}

Como ya hemos indicado, recogimos de los Cinco Libros de la parroquia de Torrellas 274 actas útiles de defunciones o entierros de personas mayores y jóvenes. Realmente, el número de actas encontrado fue de 280 , pero en seis de ellas no aparecía la fecha del entierro, por lo que finalmente dispusimos para nuestro trabajo de 139 actas de entierro de varones y de 135 actas de entierro de mujeres. Los números totales de entierros de cada año aparecen en la tabla 1. Entre los varones enterrados, 22 eran calificados como jóvenes (mozos), 25 aparecían como solteros, 3 como casados y 0 como viudos. Los 89 restantes no tenían calificación alguna. Hemos supuesto que todos eran casados. En el caso de las mujeres, 21 aparecían como jóvenes (mozas), 3 como solteras, 20 como viudas, 38 como casadas. En 57 casos se califican como "esposa de". Así pues, en 82 casos aparece algún tipo de calificación en el estado civil y en 53 casos no.

A través de un cálculo semejante al llevado a cabo en los casos de los bautismos y matrimonios, se puede también hallar "el mejor valor de entierros de mayores del año 1610" con el que calcular una TBM (Tasa Bruta de Mortalidad), en este caso de mayores, para la población de Torrellas. Esta TBM es de 10,44 entierros por mil habitantes. La figura 2 contiene la mejor recta de difuntos o entierros para el caso de Torrellas (1597 a 1609).

Como puede constatarse, en la época estudiada los entierros de mayores en Torrellas, a diferencia de los bautismos y matrimonios, no fueron decreciendo al acercarse la fecha de la expulsión de los moriscos. Tal comportamiento tiene lógica, pues la muerte no es un acto voluntario por lo general, a diferencia de las bodas. Sin embargo, la pendiente de la recta de entierros de Torrellas no es elevada. Se podría decir que las pautas de mortalidad de mayores de la población de Torrellas prácticamente no cambiaron entre los años 1597 y 1609. En cambio las pautas de la población del Campo de Cariñena sí que lo hicieron y, así, entre los años 1605-1606 ésta sufrió una "crisis de mortalidad tipo medio" (en los dos años citados, la cifra de enterrados creció hasta el 1,67 de la media de los habidos en los 10 años circundantes), otra, del mismo orden, en los años 1614-1615 y una muy larga e intensa en los años 1630 y 1641$1645^{16}$, crisis que se traducen, sin duda, en la aparición de un valor elevado para su TBM. Este resulta ser de 18,97 entierros por mil habitantes.

15. M.C. ANSÓN CALVO, «Un ejemplo de demografía diferencial en Aragón», p. 58.

16. M.C. ANSÓN CALVO, «Estudio comparativo sobre la incidencia de la peste de 1652 en el campo y en la ciudad. Un ejemplo de la zona aragonesa», I Congrés Hispano-Luso-Italià de Demografía Històrica, Barcelona, 1987, p. 115, fig. 3. 


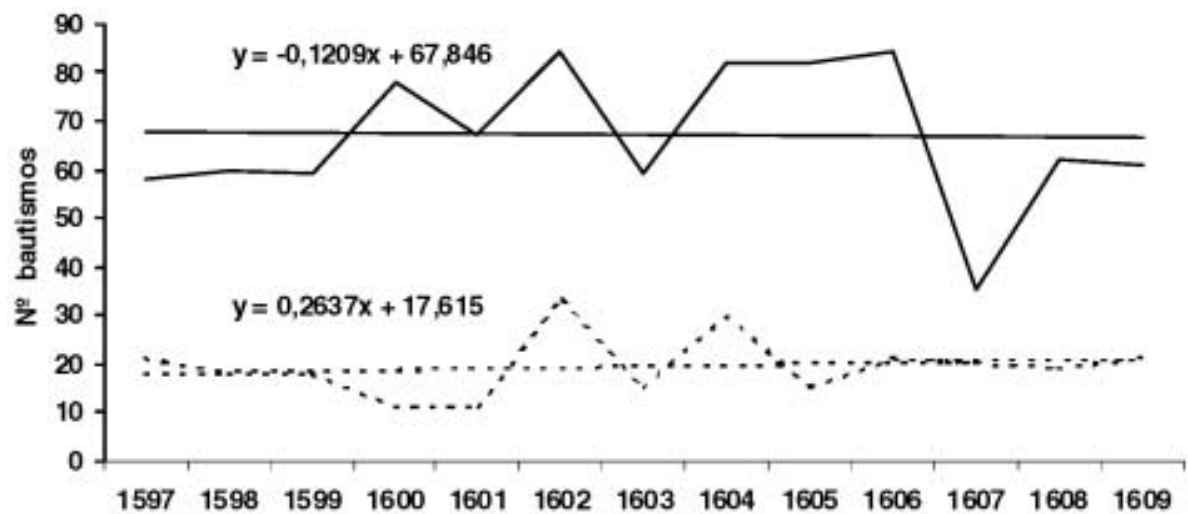

Figura 2. Números anuales de bautismos y entierros de mayores en Torrellas.

\section{RECOPILACIÓN FINAL}

Reuniendo los valores de las diferentes Tasas Brutas de Torrellas y Campo de Cariñena calculadas y referidas hasta ahora nos encontramos:

$\begin{array}{llll}\mathbf{T B N}=32,43 & \text { TBNU }=7,33 & \mathbf{T B M}=10,44 & \text { en Torrellas: } \text { datos originales } \\ \mathbf{T B N}=37,00 & \mathbf{T B N U}=9,35 & \mathbf{T B M}=18,97 & \text { en el Campo de Cariñena }\end{array}$

Curiosamente, si se dividen las TBN por las TBNU correspondientes a cada colectivo poblacional (Torrellas y Campo de Cariñena), lo que es equivalente a dividir los números promedio de ambos tipos de sucesos, bautismos y matrimonios, para una población de mil habitantes, resultan los valores de 4,42 para Torrellas y 3,96 para el Campo de Cariñena, es decir, los "hijos habidos por boda celebrada" eran un 11,61 por ciento más alto en los moriscos de Torrellas que en los cristianos viejos del Campo de Cariñena. Estos valores confirmarían que, realmente, la natalidad de los moriscos era mayor que la de los cristianos viejos. Las TBN y TBNU que, normalmente, para intervalos de tiempo no demasiado grandes se mantienen constantes, no son otra cosa que la expresión de los "comportamientos habituales de la población en relación con la natalidad y la nupcialidad" y, en cierto modo, son una medida de la fecundidad de la misma. Sin embargo, antes hemos apuntado que la nupcialidad en Torrellas quizás disminuyó al acercarse la fecha de la expulsión de su comunidad morisca. Si esto hubiera sido realmente así, la TBNU calculada por extrapolación al año 1610 de la recta de mínimos cuadrados de los números anuales de casamientos estaría, en cierto modo, contaminada y sería menor que la 
que hubiera existido en situaciones normales. Un cierto atisbo de que tal cosa pudo suceder se refleja en el hecho de que, si se divide el número total de bautismos celebrados en Torrellas en los trece años completos que fueron de 1597 a 1609 (872) por el total de bodas celebradas en los mismos años (230), resulta un valor de 3,79 bautismos por boda, es decir, un valor menor que el obtenido por división de las correspondientes Tasas Brutas de natalidad y nupcialidad. Aplicando este mismo cálculo a la población del Campo de Cariñena, resulta un valor de 3,91 bautismos por boda celebrada ${ }^{17}$, es decir, prácticamente el mismo que el obtenido por división de las correspondientes TBN y TBNU $(3,96)$ cosa que no sucede en el caso de Torrellas. Ahora bien, en el caso de la población de Torrellas, si en vez de los bautismos empíricos empleásemos los corregidos respecto a los posibles ocultamientos de los nacimientos de niñas, los 964 bautismos totales que así resultarían, divididos por las 230 matrimonios celebrados en los mismos trece años, nos conducirían a un número promedio de bautismos por boda de 4,19, valor más cercano al antes obtenido $(4,42)$ y, de nuevo, mayor que el obtenido para la población del Campo de Cariñena, lo que estaría de acuerdo con una "mayor demografía en la población de moriscos de Torrellas". Este resultado, en cierto modo, avala como cierta la suposición de los ocultamientos de nacimientos de niñas o de su mortalidad en el nacimiento.

En la figura 1 aparecen los valores de las pendientes de las mejores rectas que representan los datos empíricos de bautismos y de matrimonios de Torrellas en esta época $(-0,1209$ para los de bautismos y de -0,3901 para los de matrimonios). De estas pendientes, como se ve, la de los números anuales de bodas es mucho más negativa que la de los de bautismos. Esta situación, totalmente normal, al ser la celebración de una boda un acto voluntario y, por ello, más sometido a influencias de índole psicológico, tales como las noticias de la próxima expulsión, podría conducir, como hemos apuntado, a un valor del número anual de bodas en 1610, obtenido por extrapolación, quizás más bajo del que debiera ser. Estas consideraciones indican que, posiblemente, hemos llegado al límite de las posibilidades de los datos de partida para suministrar los "mejores valores de las TBN y TBNU de los moriscos de Torrellas", ya que disponemos de un número de valores de números anuales de sucesos pequeño $\mathrm{y}$, además, los datos proceden de un colectivo de población no muy elevada (2.040 habitantes en el año 1610). En el caso del Campo de Cariñena el colectivo humano es mucho mayor (6.111 pobladores en el año 1646) y también disponíamos de mayor número de datos correspondientes a un intervalo temporal más amplio (70 años).

La conclusión de todo este debate sobre la validez de los resultados obtenidos es que, en el caso de querer extrapolar los resultados de Torrellas a la

17. M.C. Ansón CALVO, «Un ejemplo de demografía diferencial en Aragón», p. 23, tabla 1. 
población morisca de Aragón, sería necesario ampliar el número de datos de partida, agrupando poblaciones de moriscos y recogiendo, si por fortuna existen, datos pertenecientes a un intervalo temporal más amplio en otros lugares. Por ello, estas noticias son sólo un avance de un trabajo mayor que proyectamos realizar en el futuro recogiendo datos de varios colectivos de moriscos de la misma zona a fin de mejorar los valores de estos indicadores. De cualquier modo, comparando entre sí los valores de los números promedio de bautismos por boda celebrada obtenidos sin correcciones de ningún tipo, para Torrellas $(3,79)$ y para el Campo de Cariñena $(3,91)$, se podría decir que, posiblemente, ambas poblaciones no eran demasiado diferentes en sus comportamientos demográficos respecto a la fecundidad. Sin embargo, lo que sí resulta llamativo es la diferencia entre las relaciones varón a hembra en el nacimiento para los dos colectivos, moriscos de Torrellas y cristianos viejos del Campo de Cariñena, y los valores de las TBM(M) de ambos. Respecto al valor de la $\mathrm{TBM}(\mathrm{M})$ no es posible hacer reproche alguno respecto a la correspondiente al lugar de Torrellas pues, como se ve en la figura 2, la pendiente de la recta de mínimos cuadrados es positiva. No hay, pues, indicio alguno de que la mortalidad descendiera al acercarse la fecha de la expulsión. Así pues, lo cierto es que la mortalidad de mayores en el Campo de Cariñena era 1,82 veces mayor que la de Torrellas, dato que nos parece interesante recalcar.

Antes de terminar con este capítulo de las "constantes poblacionales" del colectivo morisco de Torrellas parece oportuno hacer un comentario sobre la "mayor potencia demográfica" de los moriscos frente a los cristianos viejos en Aragón. Mediante los valores de las TBN y TBM del Campo de Cariñena, evalué en el pasado ${ }^{18}$, como diferencia entre los valores correspondientes, una "Tasa de Crecimiento Sostenido" del 7,2 por mil para este colectivo poblacional. La TBM se calculó en función del dato de que, en tres de los pueblos del Campo de Cariñena, la proporción de "entierros de niños frente al total de entierros" fue del 36,20 por ciento ${ }^{19}$. Suponiendo que en Torrellas las muertes infantiles se produjeran en la misma proporción, la diferencia entre la TBN y la TBM evaluada con esta premisa sería de 16,07 por mil habitantes, como se ve, mucho mayor (de hecho, 2,23 veces mayor) que la que presenta el Campo de Cariñena. Este resultado es, simplemente, una evaluación, pues en los libros parroquiales de Torrellas no se recogen los entierros de niños. Sin embargo, existen informaciones que, de algún modo, confirman lo hallado, respecto a la mayor tasa de crecimiento sostenido de las poblaciones de moriscos. Para obtenerlas hemos recogido los datos de población, de los años 1495 a 1787, correspondientes a 274 lugares del Valle del Ebro, de los 67 que estaban en el año 1610 poblados, prácticamente, por "sólo moriscos" (salvo el cura y su fa-

18. M.C. Ansón CALvo, «Un ejemplo de demografía diferencial en Aragón», p. 60.

19. Ibídem, p. 58. 
milia, por ejemplo), 35 por "moriscos y cristianos viejos" y 172 por "cristianos viejos únicamente". Los 67 lugares de "sólo moriscos" sumaban la población correspondiente a 2.793 "fuegos" en el año 1495. En este mismo año los moriscos que vivían mezclados en poblaciones de cristianos viejos sumaban 1.401 "fuegos", los cristianos viejos de estos mismos lugares sumaban 2.457 "fuegos" y los cristianos viejos de poblaciones de "sólo cristianos" sumaban 8.147 "fuegos" 20 . En total, en el año 1495, los cristianos computados eran los correspondientes a 10.604 fuegos y los moriscos los correspondientes a 4.194 (proporción 2,53 a 1 en el total y 1,75 a 1 en los lugares en que vivían mezclados). Entre los años 1495 a 1610 la población morisca de los 67 lugares de "sólo moriscos", si hubiera sido un conjunto aislado, hubiera tenido un crecimiento sostenido promedio de un 8,30 por mil anual y la de los 35 lugares en que vivían junto con cristianos viejos, de un 5,27 por mil anual. Como comparación, la población cristiana de los 172 lugares de "sólo cristianos viejos", entre los años 1495 a 1646, creció al ritmo de un 2,69 por mil anual.

Los valores de las tasas de crecimiento sostenido antes referidas parecen indicar que los moriscos residenciados en poblaciones en que no vivían mezclados con cristianos, tenían un crecimiento demográfico 3,09 veces mayor que la de los cristianos viejos que vivían en poblaciones de sólo cristianos. Es interesante indicar que en los casos de las poblaciones de "sólo moriscos", todas ellas pertenecían a señoríos, en su 79,10 por ciento de tipo secular. Tras la expulsión de los moriscos los señores procuraron que los lugares fueran prontamente repoblados pero, en el año 1646, su población era un 61,18 por ciento menor de la población de moriscos que tenían en el año 1610 (2.805 fuegos frente a 7.226) y sólo el 0,43 por ciento mayor de la de moriscos que tenían en el año 1495 (2.805 fuegos frente 2.793). Tenemos en este sentido algunos datos pormenorizados al respecto, en relación con los lugares de moriscos pertenecientes a los señoríos de Aranda y de Hijar. En el año 1609 el Condado de Aranda en Aragón (15 lugares de los que 12 eran de "sólo moriscos"), perdió el 72,08 por ciento de su población y en el año 1646 la población del mismo era sólo el 53,70 por ciento de la que tenía en el año 1609 y la población del Ducado de Híjar en Aragón (8 lugares, 3 de "sólo moriscos"), era el 80,08 por ciento de la que tenía en el año $1609^{21}$.

Volviendo al tema principal, con los datos aducidos podríamos concluir que, en conjunto, realmente la población de los 67 lugares de sólo moriscos tuvo un crecimiento muy bajo entre 1610 y 1646 . Aceptando el crecimiento de

20. A. Ubieto Arteta, Historia de Aragón: los pueblos y los despoblados, tomos 4 y 5.

21. M.C. Ansón CAlvo y S. GÓMEZ, «Repercusiones demográfico-económicas de la expulsión de los moriscos en el Señorío de Aranda», Actes du Ve Symposium International d' Etudes Morisques sur: Le V Centenaire de la chute de Grenade, 1492-1992, Zaghouan (Túnez), 1993, p. 115; ID., «Repercusiones demográfico-económicas de la expulsión de los moriscos en el Ducado de Híjar», Actes du Ve Symposium International d'Etudes Morisques sur: Le V Centenaire de la chute de Grenade, 1492-1992, Zaghouan (Túnez), p. 89. 
0,43 por mil anual, o sea, aceptando que la cantidad de "repobladores" fue prácticamente igual a la de moriscos que había en el año 1495, es decir, 1.401, la población cristiana de los citados 35 lugares en que vivían "etnias mezcladas", entre 1495 y 1646 hubieran crecido con un crecimiento sostenido del 2,86 por mil anual, es decir, prácticamente lo mismo que la de "sólo cristianos viejos" de los 172 lugares computados. Por otro lado, cuando ambas etnias vivían mezcladas los moriscos parece que "crecían menos que cuando vivían solos", 5,27 por mil anual frente a 8,30. De los 35 lugares implicados de este tipo, sólo 4 eran de realengo (Barbastro, Borja, Calatorao y Fraga) y los 31 restantes de señorío y, de ellos, sólo dos tenían en el año 1646 una población superior a los 2.000 habitantes. De los lugares en los que vivían sólo moriscos (67 lugares), en su 94,03 por ciento tenían una población en el año 1610 inferior a 2.000 habitantes. Los tres que la tenían superior eran Urrea de Gaén, La Puebla de Híjar, y Torrellas. Calanda bordeaba los 2.000 habitantes. Sólo en un lugar de etnias mezcladas la población de moriscos expulsados fue superior a 1.500 habitantes, este lugar fue Belchite, curiosamente "propiedad" del mismo señor secular que La Puebla de Híjar y Urrea de Gaén. Torrellas lo era del duque de Villahermosa y conde de Ribagorza. Calanda era propiedad de las Órdenes Militares.

Lo interesante de toda esta relación es que parece ser que el tamaño del lugar donde vivían influía en las tasas de crecimiento anual de las poblaciones de moriscos entre los años 1495 y 1610, pues de las 67 poblaciones de las que se expulsaron moriscos en 1610, en 50 de ellas vivían menos de 500 habitantes y su crecimiento sostenido resultó ser, en promedio, del 4,10 por mil. En 14 vivían en la fecha de la expulsión entre 500 y 1.000 habitantes y estos, entre 1495 y 1610, crecieron al 8,20 por mil. En seis vivían entre 1.000 y 1.500 habitantes y sus poblaciones habían crecido en el mismo lapso temporal al 8,74 por mil anual. En 4 lugares, en los que vivían entre 1.500 y 2.000 habitantes, lo habían hecho al 10,64 por mil, mientras que en tres, de población entre 2.000 y 2.500 habitantes en el año 1610, lo hicieron al 12,85 por mil en promedio (Torrellas era una de estas). En los lugares de moriscos mezclados con cristianos viejos, sólo en cuatro de ellos crecieron con crecimientos sostenidos del mismo orden. Estos fueron: Hijar, Pedrola, Samper de Calanda y Caspe, que en conjunto lo hicieron al 11,78 por mil anual. Curiosamente, uno de ellos, Pedrola, era propiedad del duque de Villahermosa e Híjar y Belchite pertenecían al duque de Híjar. Los moriscos que habitaban en Belchite habían crecido, entre 1495 y 1610, el 8,21 por mil anual. Estas y otras noticias extraídas de documentos de muy distinta tipología ${ }^{22}$ nos llevan a confirmar una vez más los "privilegios" que parece tenían los mo-

22. M.C. Ansón CALVO, «Almonacid de la Sierra: Un pueblo de moriscos en la encrucijada de la Expulsión», Destierros Aragoneses, Zaragoza, 1988, pp. 308-309; ID., «La convivencia día a día en una comunidad morisca aragonesa (Siglos XVI-XVII)», Actes du VIII Symposium International d'Etudes Morisques sobre: Presencia y vida cotidiana morisca en el Mediterráneo y la América Latina, 7-11 mayo Zaghouan, en prensa. 
riscos en algunos lugares de Aragón pertenecientes a señoríos seculares y entre los que podemos citar a los que ocupaban las tierras del conde de Aranda, del duque de Hijar y del duque de Villahermosa, que eran "excelentes señores de moriscos" y cuidaban de su población, lo que sin duda propició una demografía positiva a lo largo de los años. Ello se refleja en los datos procedentes de documentos notariales e inquisitoriales, amén de los Memoriales de quejas que algunos de estos señores presentaron a la Corona tras la expulsión ${ }^{23}$.

\section{RECONSTRUCCIÓN DE FAMILIAS}

El objetivo más importante de cualquier estudio de demografía histórica es llegar a reconstruir familias, ya que con ellas se pueden obtener informaciones demográficas muy importantes de las que, en principio, quizás en nuestro caso podríamos conseguir dos: el número promedio de hijos habidos por familia y los valores de los intervalos entre nacimientos sucesivos. Estas dos informaciones nos van a proporcionar también un atisbo de las posibilidades de regeneración de la población. La reconstrucción de familias pretendimos hacerla de la forma más automática posible, utilizando las posibilidades del programa de base de datos antes nombrado, recurriendo sólo a la comparación "de visu" de listados nominativos cuando no fuimos capaces de hacerlo de otro modo.

Como ya hemos descrito, disponemos de tres archivos: Bautismos, Matrimonios y Entierros o Difuntos en los que hemos vertido 966 actas de bautismos, 266 actas de matrimonios y 280 actas de entierros de mayores, respectivamente. La "acta tipo" de Bautismos contiene 26 campos para inscribir datos, la de matrimonios 33 y la de entierros 30. Una vez depuradas las correspondientes bases de datos de bautismos y matrimonios (depurar se entiende en el sentido de corregir los errores de escritura que se pudieran haber cometido, así como homologar los datos de apellidos en el sentido de, por ejemplo, suprimir los calificativos "de" poniendo, por ejemplo, Gali en vez de "de Gali", o en el de escribir siempre con b o v todos los apellidos que llevasen estas letras, etc.), en la de matrimonios cambiamos los nombres de los campos: Nombre del Novio, Apellido del Novio, Nombre de la Novia y Apellido de la Novia por los de Nombre del Padre, Apellido $1^{\circ}$, Nombre de la Madre y Apellido $2^{\circ}$ y el de Estado Civil del Novio por Nombre $1^{\circ}$. Posteriormente, en esta base de datos de matrimonios modificada, introdujimos en el ahora campo Nombre $1^{\circ}$ la palabra AAA. Después, desde el archivo de bautismos importamos los ocho datos de los campos con los mismos nombres (Apellido $1^{\circ}$, Ape-

23. M.C. Ansón CAlvo y S. GÓmEZ, «Repercusiones demográfico-económicas de la expulsión de los moriscos en el Señorío de Aranda», p. 90; y «Repercusiones demográfico-económicas de la expulsión de los moriscos en el Ducado de Híjar», p. 90. 
llido $2^{\circ}$, Nombre $1^{\circ}$, Nombre del Padre, Nombre de la Madre, Día, Mes y Año) de las 266 actas de matrimonios, creando así una especie de archivo común Bautizos-Matrimonios. En este archivo las bodas aparecen como un hijo más con nombre AAA. A partir de este archivo, la reconstrucción de familias la realizamos utilizando la facilidad "búsqueda de múltiples" para los cuatro datos de Apellido $1^{\circ}$, Apellido $2^{\circ}$, Nombre del Padre y Nombre de la Madre ya que, dentro de ellos, tienen que estar las bodas e hijos correspondientes. Como es lógico, dado que el número de apellidos que llevaban los pobladores de Torrellas no era excesivamente variado, nos aparecieron algunos matrimonios que no tuvieron hijos bautizados en Torrellas y, también, grupos de hijos para los que no constan los matrimonios de sus padres por haberse celebrado antes del comienzo del primer libro parroquial de Actas Sacramentales encontrado, pero lo que sí es cierto es que aquellos matrimonios que no aparecieron se debió, con seguridad, a que no tuvieron hijos bautizados en Torrellas y tampoco aparecieron los bautizados que eran hijos únicos de matrimonios no celebrados en Torrellas.

Una vez llevado a cabo todo el procedimiento, nos quedaron como "familias iniciadas pero no terminadas a causa del evento de la expulsión", 135 familias (56 con un hijo encontrado, 46 con dos hijos, 17 con tres hijos, 14 con 4 hijos y, finalmente, 2 con 5 hijos), familias que tuvieron entre los años 1596 y 1610, 265 hijos, es decir, en promedio, 1,96 hijos por familia. Este valor es muy inferior al obtenido por división entre el total de bautizos y el total de bodas (entre los años 1597 y 1609), valor que, como vimos era de 3,79. En el Campo de Cariñena, entre los años 1620 y 1650 (30 años) 238 "familias iniciadas y terminadas dentro del periodo" bautizaron 842 hijos, es decir, un promedio de 3,54 por familia. Ahora bien, no podemos comparar entre sí estos datos de Torrellas y Cariñena $(1,96$ y 3,54) ya que los periodos de observación son diferentes y también lo es la tipología de las familias halladas. Ello conduce sin duda a que la probabilidad de que aparezcan familias con un hijo es mucho mayor en el caso de Torrellas que en el del Campo de Cariñena. Así, en Torrellas el 41,48 por ciento de las familias encontradas tuvo "1 hijo" mientras que en el Campo de Cariñena sólo lo fue el 21,85 por ciento, a pesar de ser doble el periodo de observación en este último caso. Sin embargo creemos que, probablemente, más que la amplitud del periodo de observación, influye en las diferencias halladas el hecho de ser familias terminadas las computadas en el caso del Campo de Cariñena.

Para encontrar en el caso de Torrellas posibles familias terminadas mezclamos los archivos de matrimonios y de entierros y, para hacerlo, seguimos un método semejante al anteriormente descrito para mezclar los de bautismos y matrimonios. Para ello, una vez depurado el archivo de entierros, incluyendo en la depuración la eliminación de los entierros de "jóvenes" (mozos y mozas) y los que aparecía el individuo como "soltero(a)", cambiamos los nombres de los "campos correspondientes a la filiación del enterrado" por la terminología correspondiente al archivo de bautismos (Apellido del Difunto, si es varón 
por Apellido $1^{\circ}$, y por Apellido $2^{\circ}$ si es hembra y, lo mismo, en el caso de los Nombres Propios correspondientes, por Nombre del Padre y Nombre de la Madre). Además, el campo de "Licencia" lo transformamos en Nombre $1^{\circ}$. En este campo, incluimos ZXY si el difunto era varón y ZXX si era hembra. Con ello, prácticamente en el 90 por ciento de los casos, caracterizamos a los difuntos. Nos quedaron sin embargo algunos casos, aquellos entierros de mujeres en que ponía "la mujer de...", casos que, en su mayor parte, se resolvieron a través de mezclar los archivos de matrimonios y de entierros de mujeres, una vez hechos los cambios adecuados en los nombres de los campos del archivo de entierros. Obtuvimos así un archivo de entierros de "casados" que contenía, prácticamente, casi todos los entierros, pues los pocos que nos quedaron sin filiación se debió a que el párroco sólo los identificó por el "mote" y no por su nombre y apellido. Una vez llevada a cabo toda esta tarea, desde el archivo de bautismos importamos el archivo de entierros más el de matrimonios modificados, con lo que obtuvimos un archivo final de bautismos, matrimonios y entierros. Ordenándolo por apellidos encontramos 16 familias "iniciadas y terminadas" ( 4 de 1 hijo, 6 de 2 hijos, 4 de tres hijos y 2 de 4 hijos) que tuvieron en total 52 hijos, con lo que el número promedio de hijos por familia para este colectivo resultó ser de 3,25, como se ve mucho más próximo al hallado para el Campo de Cariñena de 3,54. En este último caso, el número de familias encontradas con 1 hijo representa sólo el 25 por ciento, valor mucho más próximo al antes citado para el Campo de Cariñena (21,85 por ciento). En adición a este argumento, como veremos después, si las dos familias de 5 hijos, iniciadas y no terminadas, que habíamos hallado hubieran sido del "tipo terminado", el número de hijos promedio hallados para las 18 familias implicadas hubiera sido de 3,44. Las dos familias encontradas que tuvieron cinco hijos dentro del periodo de observación tuvieron su último hijo en el año 1609 (febrero y mayo, respectivamente), lo que, unido al intervalo más probable entre nacimientos sucesivos, conduce a que aunque hubieran tenido después uno, o más hijos, no los hubiéramos podido encontrar.

Aunque no ha sido a través de la reconstrucción de familias, podemos aportar algún dato nuevo sobre el "número promedio de hijos por familia", en este caso, de familias en las que, realmente, vivían los hijos habidos. Una survey de los testamentos recogidos en los Protocolos de los Notarios de Torrellas entre los años 1564 y 1606, testamentos en los que aparecen los hijos vivos de los otorgantes, nos ha permitido encontrar el número de hijos que tenían 41 familias. De ellas, 1 tenía un solo hijo, 5 tenían dos hijos, 11 tenían 3 hijos, 15 tenían 4 hijos, 5 tenían 5 hijos, 2 tenían 6 hijos, 1 tenía 7 hijos y 1 tenía 9 hijos. En conjunto las familias en las que el padre testó tenían 157 hijos vivos en el momento del testamento, lo que conduce a que, en promedio, tenían 3,83 hijos por familia. Respecto a este dato no podemos decir que, dado el pequeño intervalo temporal en que aparecen (42 años), la probabilidad de que aparecieran más las familias con pocos hijos fuera elevada. Sin embargo sí que se podría hacer el reproche de que las familias implicadas eran, quizás, las "ricas" del 
lugar. De cualquier modo, lo que sí resulta claro es que la cifra hallada, 3,83 hijos por familia, no se puede comparar con la antes obtenida para Torrellas de 3,25 hijos habidos por familia terminada, pues la primera da los hijos vivos de la familia y la segunda los hijos que le nacieron, de los que una cierta proporción no estaría viva cuando murió el progenitor que terminó la familia. El hecho de que la familia promedio tuviera casi 4 hijos vivos en el momento del testamento del padre indica sin embargo que, muy probablemente, estas familias generaron más de 4 hijos en promedio. Tanto el cociente entre las TBN y TBNU de Torrellas, como el obtenido por división del número total de bautismos, corregidos de la ocultación de los de niñas, por el de bodas, son mayores que 4 (4,42 y 4,19 hijos habidos por boda celebrada), lo que en cierto modo está de acuerdo con la afirmación anterior pero, al no disponer de datos directos sobre la mortalidad de niños en Torrellas, que nos permitiría determinar cuántos de los hijos habidos llegaban a una edad en la que la iglesia los consideraba "jóvenes" y los apuntaba en las actas sacramentales de entierros, sólo podemos añadir algunos datos indirectos al respecto.

Utilizando los valores de los intervalos entre nacimientos sucesivos de las familias iniciadas de Torrellas que tuvieron más de 1 hijo, se obtiene que de los 360 casos en que esto sucedió, en el 55,56 por ciento de ellas (200 casos) los hijos vivieron más de 2 años y que en el 33,06 por ciento vivieron entre un año y 2. Sólo el 11, 38 por ciento de los niños nacidos murió, probablemente, antes del año de edad. Disponemos de datos sobre la mortalidad de niños en estas edades y en esta época para la población de Zaragoza ${ }^{24}$, de los que se deduce que, antes del año de edad, morían probablemente el 18,88 de los nacidos un año antes. El comportamiento de la población de Zaragoza no es quizás el mejor para compararlo con el de Torrellas, pues Zaragoza era una ciudad grande y tenía los inconvenientes ecológicos que genera una gran acumulación de personas, pero la cifra hallada para la mortalidad infantil resulta ser mucho mayor en Zaragoza que en Torrellas. Este dato concuerda a su vez con el de que, en Zaragoza, la tasa bruta de mortalidad de mayores era mucho mayor que la de Torrellas, 18,00 por mil habitantes en Zaragoza frente a 10,44 por mil en Torrellas $^{25}$. Estas diferencias explicarían nuestra suposición de que el mayor crecimiento de las poblaciones de los moriscos se produjo quizás por el lado de una menor mortalidad, suposición que ya avancé en una publicación anterior sobre la población morisca del Ducado de Híjar ${ }^{26}$.

Estos comentarios destacan la trascendencia que tiene la fecha inicial en que en las parroquias comenzaron a recogerse de forma sistemática las Actas

24. M.C. Ansón CALVo, Demografía y sociedad urbana en la Zaragoza del siglo XVII, p. 124.

25. M.C. Ansón CALvo, «Un ejemplo de demografía diferencial en Aragón», p. 58.

26. M.C. Ansón CAlvo y S. GÓMEZ, «Repercusiones demográfico-económicas de la expulsión de los moriscos en el Ducado de Híjar», pp. 87-88. 
Sacramentales, necesarias para poder reconstruir familias y obtener datos fidedignos de los comportamientos demográficos de poblaciones de moriscos, ya que la situación real es muy complicada por el hecho de que las anotaciones de actos parroquiales referentes a moriscos terminan abruptamente en el año 1610.

\section{EDAD MÁS PROBABLE DE CASAMIENTO DE LAS MORISCAS DE TORRELLAS}

Con el intervalo temporal de 15 años (2 incompletos), intervalo en el que disponemos de datos de bautismos y de entierros, sólo sería posible, en principio, obtener, en el mejor de los casos, datos de edad de casamiento de las moriscas de Torrellas inferiores a 15 años. De cualquier modo, aunque se hallara algún caso, éste no sería significativo al respecto, pues el número de casos encontrados sería muy pequeño. Sin embargo, a fin de comprobar estas afirmaciones, hicimos una búsqueda en un archivo conjunto de mujeres y de novias y no encontramos ningún caso en el que alguna de las nacidas en los años 1596 y 1597 se casase antes de la expulsión en agosto de 1610.

Aznar Cardona relata: "que se casaban a los 11 y 12 años y su fuerte atracción al pecado de la carne", "engendraban como conejas teniendo sus casas bullendo de hijos como hormigueros, a los que no podían alimentar" ${ }^{27}$. Casey, citado por Domínguez Ortiz y Vincent ${ }^{28}$, mediante reconstrucción de familias, obtiene como edad media de casamiento en un parroquia del Reino de Valencia, Pedralba, 18 años para las moriscas y 20 para las cristianas viejas. Vincent, por su parte ${ }^{29}$, a partir de los recuentos de moriscos de Córdoba en 1572 y de Extremadura en 1594, establece la edad aproximada de matrimonio de las moriscas en una media de 18 a 19 años, tras un sondeo de 291 casos y empleando un método que se basa en restar de la edad de la mujer casada la edad de su primer hijo (¿el hijo más pequeño?) aumentada en un año por el intervalo entre la boda y el nacimiento de este hijo.

El mismo Vincent ${ }^{30}$ utilizando 210 declaraciones ante la Inquisición de mujeres moriscas de Carlet, de ellas 164 casadas (78,10 por ciento del total), de las que la más joven tenía 11 años de edad, llega a la conclusión de que el matrimonio de las moriscas es precoz, generalizadamente. En la tabla 1 de su trabajo presenta los datos de Carlet referentes a las mujeres casadas y solteras

27. P. Aznar Cardona, Expulsión justificada de los moriscos españoles, Huesca, 1612, vol. II, pp. 35 y 53.

28. A. Domínguez Ortiz y B. Vincent, Historia de los moriscos: Vida y tragedia de una minoría, Madrid, 1978, p. 85.

29. Ibídem., p. 84 .

30. B. VINCENT, «Eléments de Démographie Morisque», La Corona de Aragón y el Mediterráneo, Siglos XV-XVI, Zaragoza, 1997, p. 152. 
hasta los 25 años de edad de las declarantes ( 93 casos en total, 48 de casadas y 45 de solteras). Trabajando los datos de la tabla citada de Vincent obtuvimos la figura 3, que refleja la distribución, en función de los años, de los datos de edades de moriscas de Carlet $^{31}$.

Esta gráfica contiene las "mejores representaciones poli-nómicas de los datos", de las que se podría decir que conducen a una edad de 18,50 años como "mejor edad en que los números de solteras igualan a los de casadas". Ahora bien, este resultado es una consecuencia de que se omiten, o no las había, casadas de edades superiores a 25 años, lo que le da cierto peso a las edades de casadas inferiores a los 20 años. De las curvas de la figura 3 lo que sí resulta claro es que solteras con edades superiores a 20 años prácticamente no aparecen, pero el peso de las casadas de más de 25 años no se puede saber cuál es. Si los datos de edades de casadas superiores a 25 años hubieran aparecido, la curva de casadas quizás se hubiera desplazado a la derecha y "el corte" con la curva de solteras podría haber aparecido en 19 años, o en quizá más.

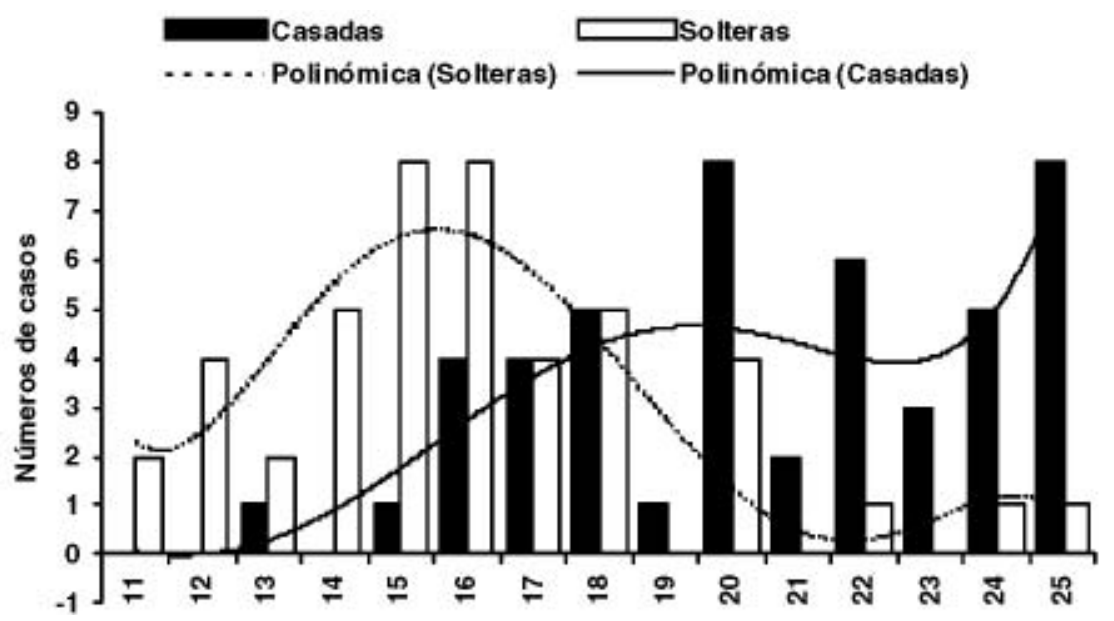

Figura 3. Distribución por edades de mujeres moriscas, casadas y solteras, en Carlet.

Por otro lado, a través de los procesos inquisitoriales de aragoneses de los años 1579 a 1609 (A.H.N.: Legajos 988, 989, 990 y 991) que hemos estudiado, encontramos las edades de 85 mujeres casadas encausadas, edades que se

31. Ibídem. 
distribuyen desde los 16 a los 70 años, aunque mayores de 50 años sólo aparecen 2, una de 54 años y otra de 70 años. La figura 4 pone de manifiesto la distribución por edades de los datos encontrados.

La primera precisión que hay que hacer en relación con ellos es que la aparición de 13 casos de casadas de 30 años y otras 13 de 40 años indica, muy probablemente, que corresponden a moriscas cuya capacidad de manifestar su verdadera edad era muy limitada y que, en relación con este aspecto de su filiación, sólo conocían aproximadamente su edad. Ciertamente, entre las edades de 30 años (13 casos) y las de 40 años (13 casos), aparecen 12 casos en que se recogen las edades con mucha más precisión pero, muy probablemente, sucedió realmente lo que acabamos de indicar en los casos de las que decían ser de 30 y 40 años justos.

Hasta los 29 años, se recogen en esta encuesta 39 casos (45,88 por ciento del total). De los 30 a los 49 años aparecen 40 casos (47,06 por ciento del total) y de los 50 en adelante 6 casos (7,06 por ciento del total). La edad promedio de las 85 moriscas casadas encontradas resulta ser de 31,87 años, la de las 39 halladas de menos de 30 años, de 24,62 años, la de las 40 que tenían edades entre 30 y 49 años de 35,63 y la de las 6, de edades entre 50 y 70 años, de 54 años. Eliminando los seis casos de más de 50 años, resulta una edad promedio para las 79 moriscas casadas así encontradas de 30,19 años, es decir, un valor no mucho menor que el que se obtiene con las edades de las 85 moriscas casadas encontradas. Por otro lado, moriscas solteras en las que constase su edad hemos encontrado sólo un caso, aunque en otras 11, al no constar el nombre del marido, hemos supuesto que eran solteras. Estas 12 "solteras", ninguna de edad superior a los 28 años, conducen a una edad promedio de 18,17 años.

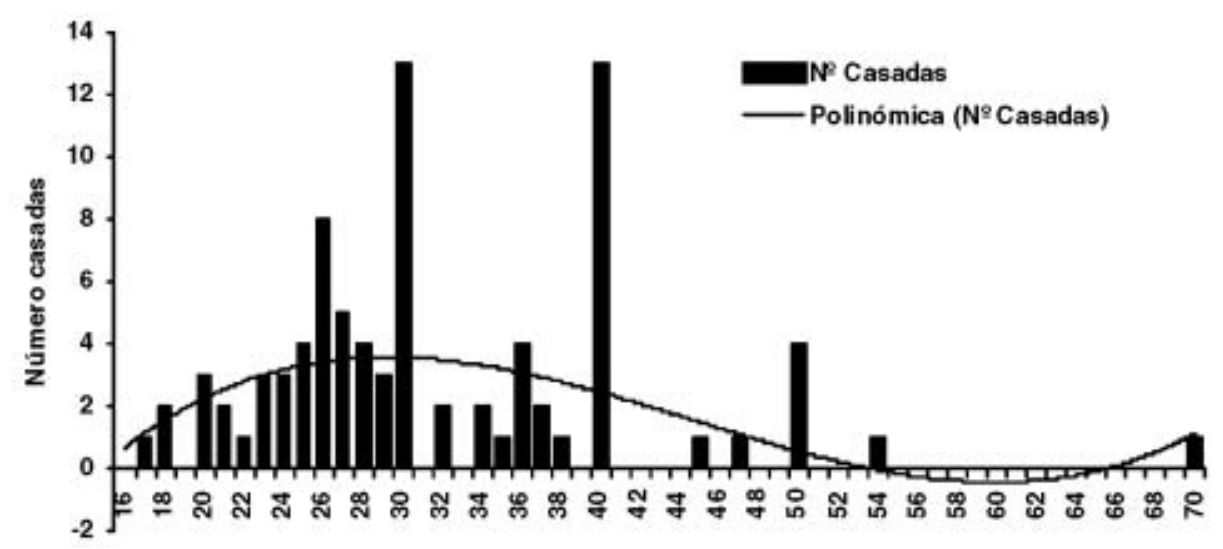

Figura 4. Número de casados en función de la edad. 
Con estos datos no es posible obtener mejores aproximaciones al valor

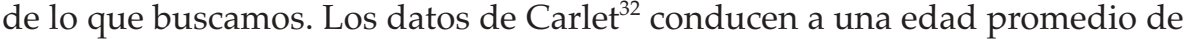
las casadas de 19,45 años y los que acabamos de reseñar a otra de 31,87, como se ve, muy diferente de la anterior. Ninguna de las dos puede permitir un cálculo de "la edad más probable de casamiento de las moriscas", pero la últimamente reseñada no está a favor, ni mucho menos, de la idea de que las moriscas se casaban "a edades muy tempranas". Ahora bien, todos los datos encontrados no forman un "censo de población" y, además, los datos de los censos que son útiles para calcular la "singulate mean age at marriage, o SMAM $^{\prime \prime 33}$ son los correspondientes a las cohortes de solteras en el caso de las mujeres y, de estas, hemos hallado muy pocas para que los valores de sus edades sean útiles para cálculos más elaborados.

Parece, pues, que los únicos datos que podrían aportar algo al problema de si la "elevada tasa de crecimiento de las poblaciones de los moriscos provenía de la temprana edad de casamiento de las moriscas", son los datos parroquiales de bautismos y de casamientos de las moriscas implicadas. A este respecto existen los datos de Maíso y Blasco ${ }^{34}$. Con sólo 7 moriscas del lugar de Fréscano cifran la edad promedio de casamiento en 18,13 años. Los datos de bautismos y matrimonios que manejan estos autores se extienden desde el año 1583 al año 1609 (27 años). Ciertamente, en este intervalo temporal podrían haber hallado que se casa alguna morisca de 26 años pero, dado el muy pequeño tamaño de la población del pueblo (450 habitantes), la probabilidad de que alguna de las aproximadamente 7 niñas nacidas en el año 1583, llegase a casarse en una de las 5 bodas celebradas en el año 1609, es muy pequeña y está claro que la probabilidad de encontrar novias de menos años crece al disminuir la edad de casamiento. Así pues, el promedio encontrado es muy circunstancial y, dependiendo de causas aleatorias, podría ser mayor o menor. De cualquier modo, el valor coincide bien con el antes citado de Casey para las moriscas de Pedralba (18 años), con el ya citado de Domínguez Ortiz y Vincent (entre 18 y 19 años), con el que antes hemos aproximado de los datos de Vincent para Carlet (18,50 años), con el obtenido como promedio de las edades de las 12 moriscas solteras halladas en las Relaciones de Causas Inquisitoriales de Aragon (18,17 años) y con el que da Pla Alberola ${ }^{35}$ de 18,08 años para Turis en Valencia. A pesar de que son "unas edades más probables de casamiento" que proceden de fuentes diversas obtenidas, además, por distintos métodos, las

32. Ibidem.

33. V. Pérez Moreda y D.S. ReHER, «Mecanismos demográficos y oscilaciones a largo plazo de la población europea (1200-1850)», Revista de Historia Económica, año IV, 3 (1986), pp. 467-490.

34. J. Maíso GonZÁlez y R.M. Blasco MartíneZ, Fréscano, 1583-1655. Una población morisca a través de los registros parroquiales, Zaragoza, 1980-1981, p. 66.

35. P.J. Pla Alberola, «Familia y matrimonio en la Valencia moderna. Apuntes para su estudio (Siglos XV-XIX)», en La Familia en la España Mediterránea, Barcelona, 1987, p. 125. 
podríamos promediar, operación que conduciría a una edad más probable de casamiento de 18,26 años.

Como comparación, el valor obtenido para la población del Campo de Cariñena, formada por más de 6.111 cristianos viejos y cuyos comportamientos estamos comparando sistemáticamente con los de la de Torrellas, es de 22,6 años ${ }^{36}$. Este dato se obtuvo como promedio de las edades de casamiento de 658 mujeres, edades que se distribuyen formando prácticamente una gausiana. Es decir, se podría considerar que las moriscas se casaban, en promedio, 4,3 años antes que las "cristianas viejas" lo cual, si en el caso de las moriscas casadas los intervalos promedio entre nacimientos sucesivos tras el primer hijo eran, como es de presumir, no muy diferentes a los hallados para las "cristianas viejas" (31,6 meses en el Campo de Cariñena), podría llevarles a tener un "número promedio de hijos por familia" superior en un hijo (5,42 hijos) respecto al de las familias "de cristianos viejos" del Campo de Cariñena (4,42 hijos por familia).

\section{INTERVALOS BODA-NACIMIENTO DEL PRIMER HIJO Y ENTRE NACIMIENTOS SUCESIVOS}

Las 135 familias para las que se conoce la fecha de iniciación permiten hallar un "valor promedio" para el intervalo entre la boda y el nacimiento del primer hijo y, sobre todo, una visión sobre la distribución temporal de este intervalo. La figura 5 representa la distribución temporal de los datos correspondientes a las 135 familias iniciadas para los que se dispone de este dato en Torrellas (1596 a 1610) y de 134 datos de familias igualmente iniciadas de un lugar del Campo de Cariñena, Aguarón (1620 a 1650), para las que, igualmente, se dispone del mismo tipo de dato. Para realizar la comparación se ha elegido Aguarón en vez de "todo el Campo de Cariñena" pues, de este modo, se comparan los comportamientos de números de datos y entidades poblacionales muy semejantes, situación que no se hubiera dado utilizando los del Campo de Cariñena.

Las distribuciones temporales de los datos recogidos en estos dos casos son muy semejantes y conducen a acumulaciones de intervalos boda-nacimiento del primer hijo prácticamente iguales, como ponen cualitativamente de manifiesto "las formas" de las líneas de tendencia polinómicas (de grado 5 en ambos casos). Cuantitativamente, en el caso de los datos de Aguarón, el 41,04 por ciento de los nacimientos se produjeron en un intervalo de 7 a 12 meses tras la boda, mientras que en el caso de Torrellas lo hicieron en el 30,08 por ciento. Esto indica que en ambos casos entre el 30 y el 40 por ciento de las parejas que se casaron tuvo su primer hijo en un intervalo temporal marcado por la fisiología. Contando con que el número de casos analizado es pequeño en ambos lugares, lo cier-

36. M.C. ANSÓN CALVO, «Un ejemplo de demografía diferencial en Aragón», p. 58. 
to es que los casamientos de mujeres embarazadas, si se entienden por tales aquellas que tuvieron su primer hijo en el intervalo de 1 a 6 meses tras la boda, suponiendo que "en todos los casos" fueron nacimientos viables y no abortos prematuros, representan el 2,99 por ciento de los bautismos en el caso de Aguarón y el 6,02 por ciento en el de Torrellas, dato que parece interesante señalar.

En la figura 5 aparecen los valores de $\mathrm{R}^{2}$ (cuadrado del coeficiente de correlación) de las dos representaciones polinómicas de los datos. Estos valores son parecidos aunque indican que la representación de los datos de Torrellas es, podríamos decir, "mejor" desde el punto de vista estadístico, sin olvidar que, ambas, son mediocres. El pequeño número de datos empleados en los dos casos es quizás la causa de este resultado.

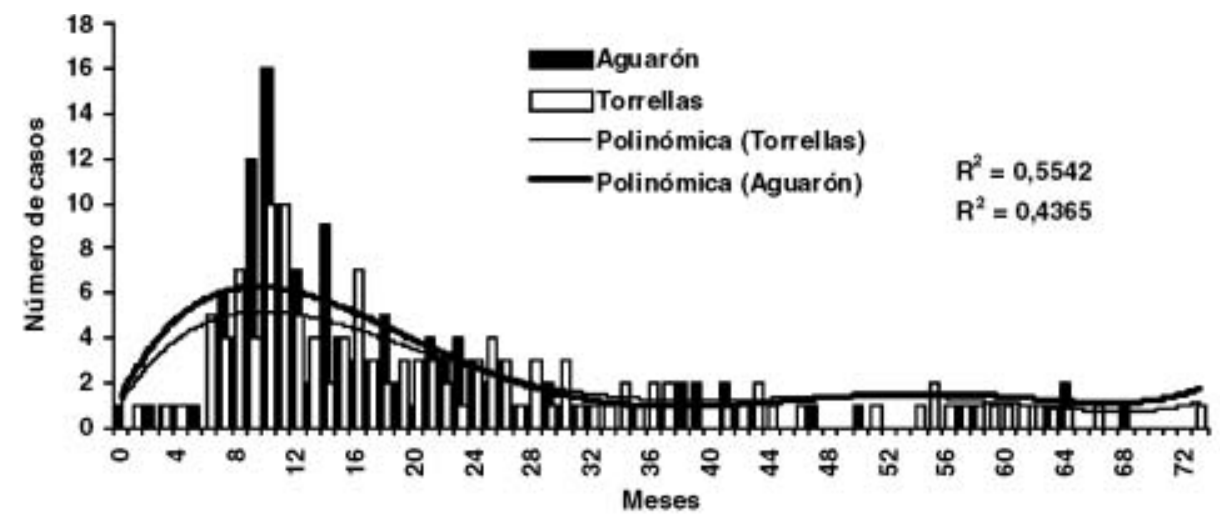

Figura 5. Intervalo, en meses, entre la boda y el nacimiento del primer hijo. Aguarón (1620-1650) y Torrellas (1596-1610).

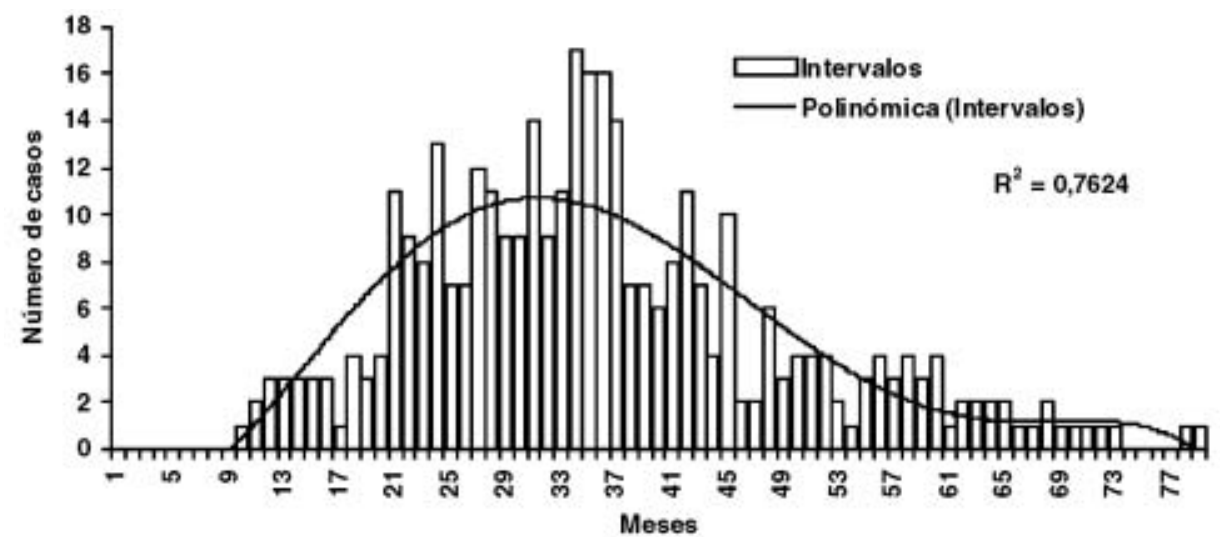

Figura 6. Intervalos entre nacimientos sucesivos en Torrellas para todas las familias con más de un hijo 1596-1610. 
Utilizando todos los casos de bautismos de hijos de familias, iniciadas o no, que tuvieron más de un hijo (579 hijos de 211 familias) es posible hallar 361 datos de intervalos temporales entre nacimientos sucesivos en el pueblo de Torrellas para los años 1596 a 1610, datos que hemos representado en la figura 6. Los 361 valores de intervalos entre nacimientos sucesivos conducen a un valor promedio de $36,84 \pm 16,46$, es decir, a un intervalo promedio de 3,07 años. Si en vez de los 361 datos procedentes de "todos" los bautismos no primerizos encontrados, consideramos sólo los 350 cuyos valores estaban entre 0 y 72 meses, el promedio se reduce a 35,07 meses. Los valores de estos promedios no tienen mayor importancia en sí mismos, pues responden a los intervalos normales entre nacimientos sucesivos en las familias de la época, pero sí que tienen valor para determinar hasta qué punto las familias moriscas y las cristianas viejas eran diferentes en sus comportamientos sobre amamantar a los hijos pequeños, toda vez que, por lo general, esta práctica acababa cuando a los niños les crecían los dientes, es decir, hacia los dos años de edad. Es interesante en este punto recordar el comentario de Mosen Yerga al hablar de la expulsión de los moriscos de Torrellas, pues denomina "niños de teta" a los de "dos años abajo".

En el tantas veces citado Campo de Cariñena, entre los años 1600 y 1650, para 3.439 casos de intervalos entre nacimientos sucesivos (también de valores de 0 a 72 meses), correspondientes a los ocho pueblos del mismo, se obtiene un valor promedio de 30,45 meses, promedio que sube a 31,57 meses si se toman los 3.581 casos encontrados ${ }^{37}$. En Torrellas, el número de intervalos superiores a 72 meses es un 3,05 por ciento del total, mientras que en el Campo de Cariñena es del 3,97 por ciento. En función de que en Torrellas se promedian "todos" los nacimientos múltiples encontrados, mientras que en el Campo de Cariñena sólo se promedian los correspondientes a familias terminadas y que, además, el Campo de Cariñena suministra diez veces más datos que Torrellas, no es evidente que la comparación de los casos de Torrellas y del Campo de Cariñena sea totalmente irreprochable. Sin embargo, obviando este inconveniente, por otra parte menor, la única conclusión posible es que ambas comunidades tenían en estos aspectos comportamientos muy parecidos aunque, en el mejor de los casos, podríamos concluir que las moriscas amamantaban durante algo más tiempo a sus hijos que las cristianas viejas. Este resultado no apoya pues la conclusión de que los matrimonios de moriscos fueran más prolíficos que los de cristianos viejos, supuestas esperanzas de vida y edades de climaterio iguales para las mujeres de ambos colectivos.

37. M.C. ANSÓN CALVO, «Un ejemplo de demografía diferencial en Aragón», p. 58. 


\section{DIFERENCIAS EN COMPORTAMIENTOS SOCIALES}

\section{NATALIDADES, NUPCIALIDADES Y MORTALIDADES ESTACIONALES}

Las figuras 7, 8 y 9 recogen las representaciones gráficas de los números mensuales de matrimonios, bautismos y entierros de mayores de los colectivos poblacionales de Torrellas y Campo de Cariñena.

De la figura 7 se deduce que los comportamientos sociales frente al matrimonio de las dos comunidades, Torrellas (moriscos) y Campo de Cariñena (cristianos viejos), eran, desde el punto de vista religioso, prácticamente iguales. Disminuciones drásticas de matrimonios en las Pascuas (marzo y abril, Pascua de Resurrección, y diciembre, Pascua de Navidad), aumentos en enero, febrero y mayo (tras las Pascuas) y, después, disminuciones acusadas en los meses de julio y agosto (recogida de las cosechas de cereales). Ahora bien, entre ambos colectivos se observa una diferencia marcada en los meses de septiembre y octubre: muchas bodas en Torrellas en septiembre (tras las cosechas de grano) y muy pocas en Cariñena, que en cambio aumentarán en noviembre. Sin duda la recogida de la vid en el Campo de Cariñena, prácticamente nula en Torrellas, puede ser la responsable de estas diferencias.

Si a finales del siglo XVI no se cultivaba la vid en Torrellas por motivos climáticos o por motivos religiosos musulmanes, es una cuestión a debatir, pero lo cierto es que, en el siglo XVIII ${ }^{38}$, en un pueblo de "sólo moriscos" muy próximo a Torrellas, Santa Cruz de Moncayo, se cultivaba la vid y se producía vino. En Torrellas no y como una prueba de ello podemos señalar que en documentos de la época, como el Libro del Justicia de Torrellas y los Protocolos de los notarios que recogen la documentación generada por los moriscos de esa comunidad (Libro del Justicia de Torrellas, A.M.T y Protocolos Notariales de A.Not Ta), no aparecen noticias sobre su cultivo. Ciertamente, el cultivo de la vid exige un tipo adecuado de terreno de secano y Torrellas era un pueblo fundamentalmente de regadío (3,78 Ha de regadío por 1 de secano frente a 2,72 por 1 en Santa Cruz de Moncayo) $)^{39}$. Quizás los repobladores cristianos que llegaron a partir del año 1611 a Santa Cruz de Moncayo conocían mejor el cultivo de la vid que los que llegaron a Torrellas, del mismo modo que conocían mejor el cultivo del trigo pues, a finales del siglo XVIII, en Santa Cruz de Moncayo se producían 2,6 cahíces de trigo por habitante mientras que en Torrellas se producían sólo $1,6^{40}$.

\footnotetext{
38. M.C. Ansón CAlvo, Tarazona en la época de la Ilustración, Zaragoza, Institución Fernando el Católico, 1977, pp. 84-86.

39. Ibídem, p. 52.

40. Ibídem.
} 


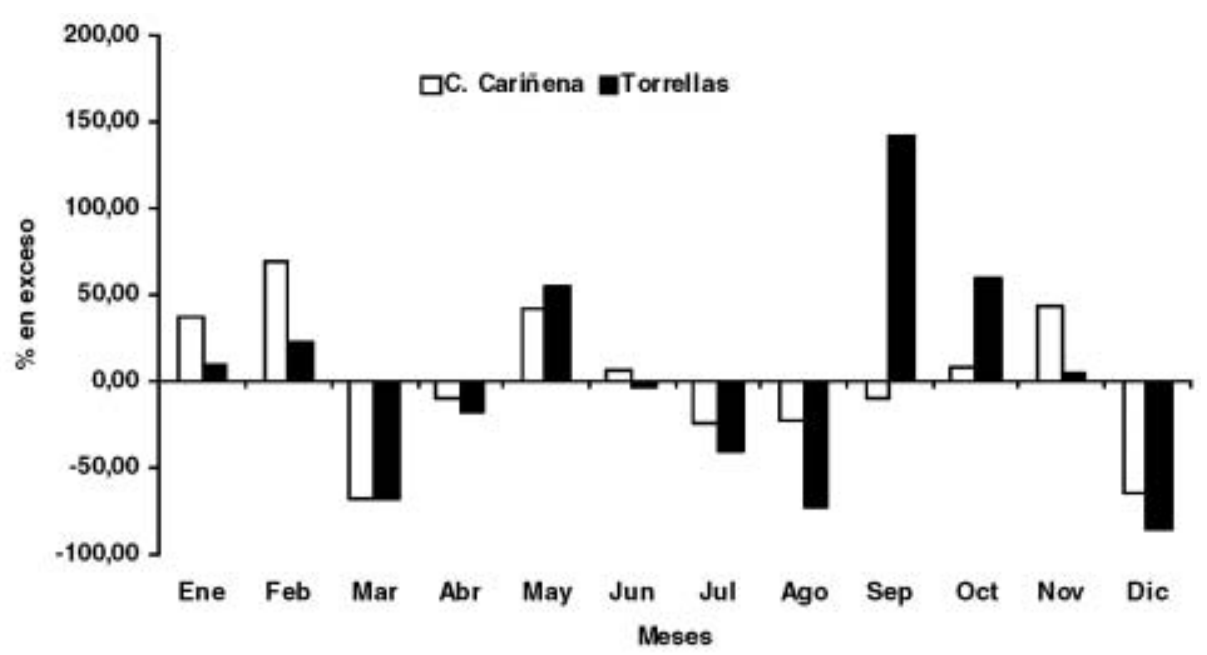

Figura 7. Número mensual de matrimonios (en \% sobre el promedio) de Torrellas y del Campo de Cariñena.

Los datos mensuales de bautismos que aparecen en la figura 8 ponen de manifiesto que durante los meses de enero, febrero y marzo (por exceso) y agosto, septiembre y diciembre (por defecto), la comunidad morisca de Torrellas y la cristiana vieja del Campo de Cariñena tenían comportamientos claramente análogos. En los meses de abril, junio y noviembre los tenían cualitativamente también análogos, excesos de nacimientos sobre el promedio en abril y defectos sobre el promedio en junio y noviembre, pero cuantitativamente diferentes y en los meses de mayo, julio y octubre los tenían contrapuestos, pues mientras en mayo en Torrellas nacían niños en exceso sobre el promedio mensual, en el Campo de Cariñena lo hacían por defecto, aunque en pequeña medida. En el mes de julio la situación es la contraria, muchos menos nacimientos que el promedio en el Campo de Cariñena y unos pocos más en Torrellas. Por su parte, en octubre, los nacimientos (bautismos) en Torrellas eran apreciablemente menos que el promedio, mientras que en el Campo de Cariñena eran, en muy pequeña medida, mayores que el promedio. En Torrellas, los excesos de bodas sobre el promedio en septiembre y octubre quizás se refleje en los excesos de bautizos de abril y mayo y el defecto de bautizos observado en noviembre quizás dependa del defecto de bodas en marzo. No podemos aportar otra explicación a lo hallado en el mes de julio que, quizás, una basada en los tipos de cultivos prioritarios en cada zona. Así, en el Campo de Cariñena, zona prioritariamente de secano, con una fuerte dedicación al cultivo de la vid, se producen muy pocos matrimonios en septiembre y octubre, con la consiguiente aparición 9-10 meses después (mayo y junio) de muy pocos niños para sumar a los que habitualmente generaban las familias ya establecidas. 
Por el contrario, en Torrellas, zona prioritariamente de regadío y sin cultivo de vid, no sucede tal cosa, especialmente en septiembre, lo que podría explicar muchas de las diferencias encontradas en este aspecto de la natalidad, en función de las necesidades que cada comunidad tenía de la fuerza de trabajo femenina como ayuda en las tareas agrarias.

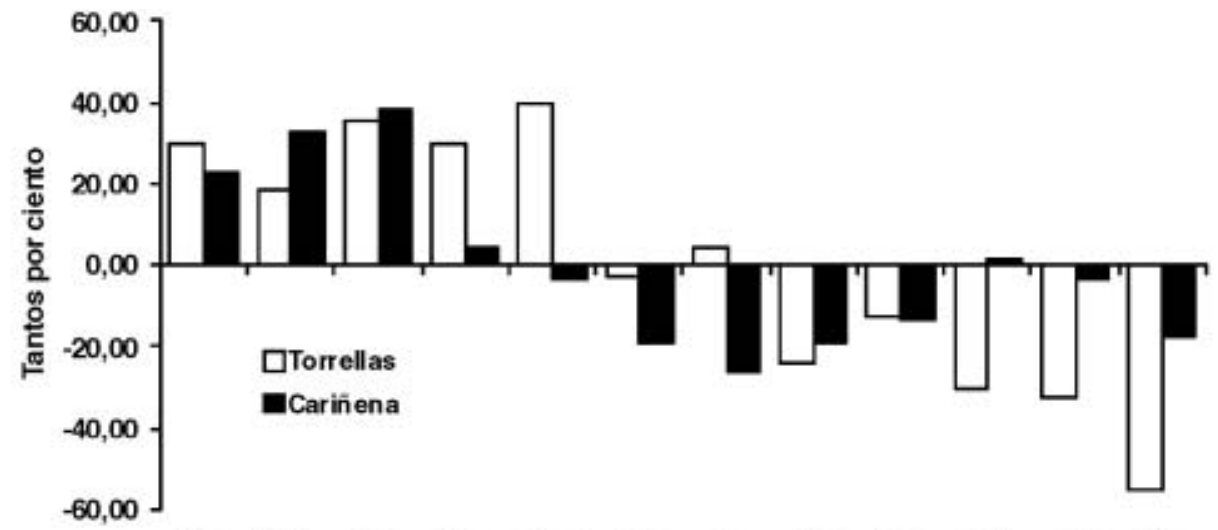

\section{Ene Feb Mar Abr May Jun Jul Ago Sep Oct Now Dic}

Figura 8. Tantos por ciento de bautismo mensuales respecto a la media de Torrellas y de Cariñena.

De la figura 9, por otra parte, se deduce que la mortalidad de mayores en el Campo de Cariñena estaba por encima del promedio en otoño e invierno (hasta enero) y por debajo del mismo en primavera y verano. Sin embargo, en Torrellas estaba por encima del promedio en enero, febrero y primavera hasta mayo y por debajo en el verano (salvo agosto) y, también, en otoño e invierno (diciembre, por ejemplo, es un mes de muy baja mortalidad). En principio se podría pensar que Torrellas, situado en el Somontano del Moncayo, tuviera un clima más frío que Cariñena y que, por ello, los meses de noviembre y diciembre podrían haber traído consigo mortalidades elevadas, sin embargo, parece que lo ocurrido es justamente lo opuesto. Por el contrario, la primavera parece como si fuera muy fría en Torrellas y mucho menos en el Campo de Cariñena. Así, todo parece llevarnos a deducir que ambas colectividades presentaban resistencias muy diferentes ante contagios de los virus habituales $y$, por ende, ante la muerte. Hay sin embargo una consideración que quizás conviene señalar. El Campo de Cariñena es una tierra de pan y vino, de secano, cosechas que, si no son nulas, proporcionan el sustento básico a la población para el año siguiente. Por el contrario, Torrellas tenía en la época y, también en 
el siglo XVIII, una agricultura mayoritariamente de huerta, de alimentos perecederos, por lo que las épocas de hambre, de haberlas, podrían estar en una estación distinta que las del Campo de Cariñena y, esto, que es diferente en distintos años, quizás pueda ser la causa de lo encontrado en Torrellas en el periodo temporal analizado.

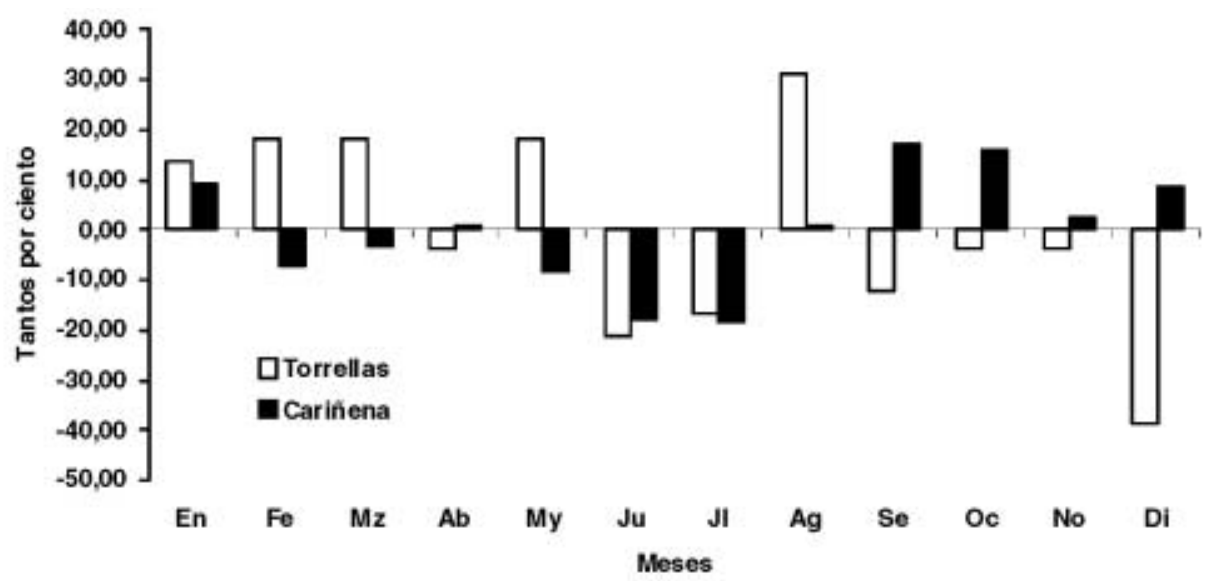

Figura 9. Excesos mensuales de entierros de mayores sobre el promedio de Torrellas y de Cariñena.

\section{CONCLUSIONES}

Tabla 2. Constantes demográficas de las poblaciones de Torrellas y Campo de Cariñena.

MES TORRELLAS TORRELLAS C. CAMP. CARIÑ. ${ }^{* *}$

Tasa Bruta de Natalidad 32,43 35,87 37,00

Tasa Bruta de Nupcialidad 7,33

9,35

Tasa Bruta de Mortalidad*

10,44

19,00

Relación varón/hembra en el Bautismo

1,32

1,06

Número de hijos por boda celebrada

3,79

4,19

3,91

Número promedio de hijos / familia

$$
-
$$

Intervalo nac. hijo $1^{\circ}$

10-11 mes.

9-10 mes.

Intervalo nac. sucesivos

3,07 años

2,63 años

SMAM

* Mortalidad sólo de mayores.

** Proceden de M.C. Ansón CALVO, «Un ejemplo de demografía diferencial en Aragón», p. 58. 
En la tabla 2 se recogen los valores de las "constantes demográficas" que hemos podido calcular para las poblaciones de Torrellas y Campo de Cariñena, a fin de posibilitar una comparación entre los comportamientos demográficos de ambas comunidades y, si es posible, encontrar una explicación al hecho de que los moriscos, durante los cinco años finales del siglo XV, todo el siglo XVI y los nueve primeros años del XVII tuvieron, en especial cuando habitaban en lugares de "sólo moriscos", un crecimiento anual sostenido de su población mucho mayor que el las poblaciones de "cristianos viejos". Como es lógico, será la concatenación de la natalidad, la nupcialidad y la mortalidad, representadas por sus correspondientes Tasas Brutas (Tasa Bruta de Natalidad (TBN), Tasa Bruta de Nupcialidad (TBNU) y Tasa Bruta de Mortalidad (TBM), las que modularán, o deberán modular, este hecho.

Si para la población de Torrellas se calculan las TBN, TBNU y TBM(M) “sin corrección alguna de los datos, se obtienen valores que, comparados con los del Campo de Cariñena, conducen al resultado de que la población de moriscos de Torrellas generaba, por mil habitantes, menos hijos (-14,09 por ciento), se casaba mucho menos (-27,56 por ciento) y sus mayores se morían menos $(-81,99$ por ciento). Las menores TBN y TBNU de los moriscos de Torrellas, en principio, están de acuerdo con el menor número promedio de hijos nacidos por boda celebrada, frente a los que se deducen para la población de "cristianos viejos" del Campo de Cariñena (-3,17 por ciento). Sin embargo, a la vista de los fuertes desequilibrios en la natalidad y la nupcialidad que hay entre ambas colectividades, lo hallado en este último aspecto de la cuestión parece que debiera haber sido mayor. En Torrellas no hemos encontrado datos sobre entierros de niños, cosa que sí hemos hallado, parcialmente al menos, en el Campo de Cariñena. Su hallazgo nos hubiera permitido calcular una Tasa Bruta de Mortalidad Total para relacionarla con la Tasa Bruta de Natalidad y calcular una Tasa Anual de Crecimiento de la Población, como hicimos en el caso del Campo de Cariñena ${ }^{41}$. Esta tasa, una simple diferencia entre las TBN y TBM(T), es en el Campo de Cariñena de 7,20 por mil habitantes nuevos que, año a año, van apareciendo en su población. Como es lógico, dadas las diferentes mortalidades relativas según la edad (Tabla de Vida), llegan a la edad de casarse y generar nuevas familias sólo 2,0128 por cada pareja ${ }^{42}$, cifra en la que no se ha tenido en cuenta los efectos de la emigración hacia Zaragoza y el celibato definitivo que, en la época, llegó a ser elevado, por la gran cantidad de fundaciones de conventos nuevos y del aumento de las cifras de religiosos en general ${ }^{43}$. Si se hubieran tenido en cuenta estos efectos, el crecimiento sería menor, estando más próximo al citado ante-

41. M.C. Ansón CALVO, «Un ejemplo de demografía diferencial en Aragón», p. 60.

42. Ibidem.

43. M.C. Ansón CALVo, «Geografía y Demografía Eclesial de España en la Edad Moderna», XIX Congreso de la Asociación de Archiveros de la Iglesia. La Laguna, septiembre 2003 (en prensa). 
riormente para las poblaciones de "cristianos viejos" (2,69 por mil anual en promedio). Ahora bien, como ya hemos comentado, la falta de una Tasa Bruta de Mortalidad de Niños (o de algún dato que nos permitiera aproximarla) en el caso de Torrellas nos impide su cálculo para su población, pero en relación con esta cuestión puede ser interesante aportar algún argumento adicional.

Anteriormente hemos señalado que no podemos calcular una SMAM para los casamientos de las mujeres de Torrellas, pero, suponiendo que fuera igual que la que parece hallarse para poblaciones de moriscas de la época en otros lugares (18,26 años), los intervalos entre boda y nacimiento del primer hijo y entre nacimientos sucesivos ( 0,875 y 3,07 años, respectivamente) harían que una mujer morisca promedio tuviera el octavo hijo a la edad de 40,63 años. Casarse muy pronto exige que las novias debieran ser, al hacerlo, fértiles. Ahora bien, ser fértiles pronto conlleva, por lo general, a edades de climaterio tempranas, y ésta es una buena razón para suponer que, difícilmente, una morisca promedio pudiera tener más de nueve hijos en total. El climaterio de las mujeres actualmente se produce, en forma variable, entre los 45 y 55 años, pero ya a los 40 años comienzan los trastornos de un estadio pre-menopáusico ${ }^{44}$. Un cálculo simple basado en la disminución del número de mujeres al ir haciéndose viejas, empleando los datos de la Tabla de Vida de la Parroquia de San Pablo de Zaragoza $^{45}$, nos lleva a que, tras ocho bautismos sucesivos, más uno inmediatamente posterior a la boda, "una morisca promedio" de Torrellas sólo podría "gestar" 4,61 hijos hasta llegar a la edad de 44 años, edad a la que muy probablemente comenzaría su menopausia. Este valor es prácticamente el mismo que el que se obtiene para una cristiana vieja promedio en el Campo de Cariñena ${ }^{46}$, capaz, hasta sus 47 años, de "poner en el mundo" 4,62 hijos. Este cálculo lo hicimos empleando, igualmente, los datos de la Tabla de Vida de Zaragoza antes citada. Lo cierto es que el hijo de más que a la "morisca promedio" le hubiera permitido gestar su temprano casamiento, lo compensan, al final, los 0,44 años de más que las moriscas daban de mamar a sus hijos frente a las cristianas viejas. Desde este punto de vista, da la impresión de que la población morisca de Torrellas y la cristiana vieja del Campo de Cariñena "tenían comportamientos muy semejantes", que habían llegado al límite de su capacidad de generar hijos y que, si ambas hubieran tenido "la misma Tabla de Vida", su crecimiento poblacional hubiera tenido que ser muy parecido. Sin embargo, los datos antes aportados sobre el crecimiento anual sostenido de las poblaciones de moriscos y de las de cristianos viejos nos dicen que algo no debió ser "igual entre ellos" y la razón fundamental de la discrepancia creemos que está en la "muy posiblemente mayor esperanza de vida de los moriscos frente a los

44. P. FARreras y C. RozMAN, Medicina interna, 1985, tomo II, p. 697.

45. M.C. Ansón CALVo, Demografía y sociedad urbana en la Zaragoza del siglo XVII, p. 119.

46. M.C. ANSÓN CALVO, «Un ejemplo de demografía diferencial en Aragón», p. 59. 
cristianos viejos". Un reflejo indirecto de ello es el menor valor de la Tasa Bruta de Mortalidad de Mayores de los moriscos de Torrellas frente a la de los cristianos viejos del Campo de Cariñena (tabla 2). En este sentido, sólo si se obtienen datos de mortalidad suficientemente representativos, si sistemáticamente aparecen para las comunidades moriscas Tasas Brutas de Mortalidad menores que las de las poblaciones de cristianos viejos, será posible comprobar esta hipótesis, hipótesis que ya avanzamos hace algunos años en otra publicación ${ }^{47}$.

Los resultados obtenidos nos llevan a concluir que, a pesar de que el pequeño intervalo temporal para el que se recogieron los datos de bautismos, matrimonios y entierros de Torrellas convierten, a veces, las evaluaciones hechas a partir de ellos en poco representativas, que los dos tipos de comunidades, Torrellas (moriscos) y Campo de Cariñena (cristianos viejos), eran "más semejantes que diferentes". De hecho, únicamente las Tasas Brutas de Mortalidad de Mayores ("no niños") calculadas para ambas comunidades son lo bastante diferentes entre sí (-81,99 por ciento en Torrellas respecto al Campo de Cariñena) para obligarnos a admitir que, muy probablemente, eran realmente distintas.

Los valores de las respectivas Tasas Brutas de Natalidad, aunque distintas, no lo son tanto (-14,09 por ciento) y además creemos que en "dar lugar" a esta diferencia pudieron actuar comportamientos "particulares" en el caso de la comunidad morisca de Torrellas. De hecho, si se corrigen los valores de números anuales de bautismos de las niñas de Torrellas de la disimetría que revelan las relaciones niño/niña, el valor corregido de su TBN (35,87 por mil habitantes) es "mucho más parecido al hallado para el Campo de Cariñena" (-3,15 por ciento). Desde luego, el valor corregido de la TBN hallado así para la población morisca de Torrellas es muy semejante al valor habitual de esta magnitud hallado en general para las poblaciones de la época ${ }^{48}$.

En el caso de las Tasas Brutas de Nupcialidad hemos encontrado una diferencia más marcada entre la correspondiente a la comunidad morisca de Torrellas frente a la de la cristiana vieja del Campo de Cariñena (-27,56 por ciento). Ahora bien, tal diferencia podría "explicarse" con razonamientos sencillos, basados en la menor cantidad relativa de moriscas solteras puestas en el mercado matrimonial, debido al menor número de "niñas nacidas" y, quizás también, en la situación anímica especial en que, seguramente, se encontraban los moriscos en las cercanías de la fecha de su"temida" expulsión.

En el trabajo «Mecanismos demográficos y oscilaciones a largo plazo de la población europea (1200-1850)» ${ }^{49}$ sus autores realizan varias afirmaciones cuya aplicación es pertinente al caso de la demografía de las poblaciones de moriscos.

47. M.C. Ansón CAlvo y S. Gómez, «Repercusiones demográfico-económicas de la expulsión de los moriscos en el Ducado de Híjar», pp. 87-88.

48. A. Domínguez Ortiz, La sociedad española en el siglo XVII, Madrid, 1967, pp. 93-96.

49. V. Pérez Moreda y D.S. ReHer, «Mecanismos demográficos y oscilaciones», pp. 476-481. 
Tras identificar España como "régimen de alta presión" en función del tipo de nupcialidad imperante, matrimonio relativamente precoz y prácticamente universal, del cual son paradigma, por otra parte, las poblaciones de moriscos, afirman, citando a Wrigley y Schofield, que "la influencia de la mortalidad sobre las tasas de crecimiento es, por lo común, mucho mayor en un sistema de alta presión que en otro de baja presión. Afirman también que, "en los sistemas de alta presión la nupcialidad, y en consecuencia la fecundidad, tienen escasas posibilidades de actuar generando un decisivo aumento de las tasas de crecimiento a largo plazo", cosa que es así precisamente "porque ya alcanzaban por lo común niveles próximos a los que permitía su máxima capacidad posible". Torrellas y el Campo de Cariñena se podrían tomar como ejemplo representativo y demostrativo de estas ideas, ya que sus mujeres hubieran llegado, en ambos casos, al mismo número promedio de hijos posibles al alcanzar el climaterio, a pesar de sus diferencias en las "edades más probables de acceso al matrimonio". Con ello, si el crecimiento de las poblaciones respectivas hubiera dependido, fundamentalmente, de la nupcialidad, el crecimiento de la población de Torrellas debiera haber sido menor que el de la población del Campo de Cariñena. Dado que sucedió todo lo contrario, la menor mortalidad de la población de Torrellas actuó y, al ser "por lo común mucho mayor la influencia de la mortalidad sobre las tasas de crecimiento", ésta resultó mayor en Torrellas que en el Campo de Cariñena, de hecho fue 5,60 veces mayor entre los años 1495 y 1646.

Finalmente, sería interesante indicar que los comportamientos diferenciales de ambas comunidades frente a la nupcialidad reflejan claramente que seguían las pautas de su condición de cristianos y las referentes a las prácticas relacionadas con la fecundidad y el cuidado de los niños (tiempo de lactancia, por ejemplo) también los asemejan bastante.

\section{RESUMEN}

Se han recogido las Actas Sacramentales de los años 1596 a 1610 del lugar de Torrellas, de población totalmente morisca, del Obispado de Tarazona en Aragón. Se han vertido en una "Base de Datos" mediante el programa comercial File Maker Pro versión 6. A través de las facilidades del programa se han obtenido las constantes demográficas, amén de otras informaciones, de su población (2.040 habitantes en el año 1610). Los resultados obtenidos se han comparado con los correspondientes a la población conjunta de varios lugares de "cristianos viejos", el Campo de Cariñena (6.111 habitantes en el año 1646). Todos los resultados del trabajo, unidos a otras informaciones procedentes de documentos y bibliografía, nos han permitido formular la hipótesis de que la causa fundamental que "hizo crecer mucho más" a lo largo del siglo XVI y comienzos del XVII la población de las comunidades moriscas, frente a las de los cristianos viejos fue, muy probablemente, la "menor mortalidad" de los moriscos. 
Palabras clave: moriscos, demografía, Torrellas, actas sacramentales, mortalidad.

\section{ABSTRACT}

We have gathered the Actas Sacramentales of the year 1596 to 1610 of the place of Torrellas, a place with totally Moorish population, of Tarazona's Bishopric in Aragon. The data has been putted in a database by means of the commercial program File Maker Pro version 6. Due to the possibilities of this we have obtained the demographic constants and other information of its population (2040 inhabitants in 1610). The results have been compared with the equivalents to the joint population of several places of "old Christians": Cariñena's Field (6111 inhabitants in 1646). All the results of the work, joined other information proceeding from documents and bibliography, have allowed to formulate this hypothesis: the fundamental reason that "made grow much more" throughout the XVIth century and beginning of the XVIIth the population of the Moorish communities, with respect too those of the old Christians was, very probably, the "minor mortality" of the Moriscos. tality.

Key words: moriscos, demography, Torrellas, actas sacramentales, mor- 